\title{
Immunthrombozytopenie - Onkopedia-Leitlinien Update: Empfehlungen einer gemeinsamen Arbeitsgruppe der DGHO, ÖGHO, SGH + SSH und GPOH
}

\author{
Axel Matzdorff ${ }^{a}$ Wolfgang Eberl ${ }^{b} \quad$ Aristoteles Giagounidis ${ }^{c}$ Paul Imbach $^{d} \quad$ Ingrid Pabinger $^{\mathrm{e}}$ \\ Bernhard Wörmann ${ }^{\mathrm{f}, g}$ \\ ${ }^{a}$ Klinik für Hämatologie und Onkologie, Caritasklinikum Saarbrücken St. Theresia, \\ ${ }^{\mathrm{b}}$ Klinik für Kinder- und Jugendmedizin, Klinikum Braunschweig, \\ ${ }^{c}$ Klinik für Hämatologie und Onkologie, Marien Hospital Düsseldorf, Deutschland \\ ${ }^{d}$ Pädiatrische Onkologie und Hämatologie, Universitätskinderspital beider Basel, Schweiz \\ ${ }^{\text {e } U n i v e r s i t a ̈ t s k l i n i k ~ f u ̈ r ~ I n n e r e ~ M e d i z i n ~ I, ~ K l i n i s c h e ~ A b t e i l u n g ~ f u ̈ r ~ H a ̈ m a t o l o g i e ~ u n d ~ H a ̈ m o s t a s e o l o g i e, ~ M e d i z i n i s c h e ~ U n i v e r s i t a ̈ t ~ W i e n, ~}$ \\ Österreich \\ ${ }^{f}$ Deutsche Gesellschaft für Hämatologie und Onkologie, Berolinahaus, \\ ${ }^{g}$ Ambulantes Gesundheitszentrum der Charité Universitätsmedizin Berlin, Campus Virchow Klinikum, \\ Medizinische Klinik mit Schwerpunkt Hämatologie, Onkologie und Tumorimmunologie, Berlin, Deutschland
}

\section{Einleitung}

Dies ist die Aktualisierung der Immunthrombozytopenie (ITP)-Leitlinien aus dem Jahre 2010 [1] durch eine gemeinsame Expertengruppe der Deutschen (DGHO), Österreichischen (ÖGHO) und Schweizerischen Gesellschaft für Hämatologie $(\mathrm{SGH}+\mathrm{SSH})$ und Medizinische Onkologie sowie der Gesellschaft für Pädiatrische Onkologie und Hämatologie $(\mathrm{GPOH})$. Sie basiert auf den bisherigen Empfehlungen der genannten Fachgesellschaften [1-3], dem Internationalen Consensus-Report [4], der American Society of Hematology (ASH)-Leitlinie [5] und der Leitlinie Immunthrombozytopenie im Kindes- und Jugendalter der Arbeitsgemeinschaft der Wissenschaftlichen Medizinischen Fachgesellschaften (AWMF) [6]. In dieser Aktualisierung werden alle relevanten Publikationen bis August 2013 berücksichtigt.

\section{Definition}

Die primäre ITP ist eine erworbene Thrombozytopenie. Das Akronym ITP steht für Immunthrombozytopenie, die Bezeichnung idiopathische thrombozytopenische Purpura sollte nicht mehr verwendet werden [7]. Ein weit verbreitetes Eponym ist der Begriff Morbus Werlhof. Man unterscheidet die primäre Form, bei der keine auslösende Ursache für die ITP erkennbar ist, von den sekundären Formen, bei denen die ITP durch Medikamente oder andere Erkrankungen ausgelöst wird (Tab. 1) [8].

\section{KARGER}

Fax +497614520714

Information@Karger.com

www.karger.com

\section{(c) 2014 S. Karger GmbH, Freiburg}

2296-5270/14/3714-0006\$39.50/0

Accessible online at:

www.karger.com/ort
Die folgenden Ausführungen beziehen sich auf die Pathophysiologie, Diagnostik und Therapie der primären ITP.

\section{Epidemiologie}

Bei Erwachsenen liegt die ITP-Inzidenz zwischen 0,2 und 0,4 Neuerkrankungen pro 10000 pro Jahr und die Prävalenz bei etwa 2 chronischen ITP-Patienten pro 10000 Personen. Bei Kindern und Jugendlichen beträgt die ITP-Inzidenz ebenfalls etwa 0,3 pro 10000 pro Jahr. Da die chronische ITP in der Pädiatrie selten ist, gibt es dazu keine Prävalenzangaben.

\section{Pathophysiologie}

\section{Vermehrter Thrombozytenabbau}

Die ITP ist eine Autoimmunerkrankung [8]. Autoantikörper gegen Thrombozyten führen dazu, dass diese von Makrophagen und dendritischen Zellen z.B. in der Milz aufgenommen und abgebaut werden.

\section{Hemmung der Thrombozytopoese}

Die Autoantikörper gegen Thrombozyten können sich aber auch gegen ähnliche Antigene auf Megakaryozyten richten und die Thrombozytopoese im Knochenmark hemmen $[9,10]$. Deshalb ist bei einigen Patienten nicht nur die Überlebenszeit der zirkulierenden Thrombozyten stark verkürzt, sondern auch die Thrombozytopoese im Knochenmark vermindert. 
Tab. 1. Einteilung der Immunthrombozytopenien und Verbrauchsthrombozytopenien anderer

Genese

\begin{tabular}{|c|c|}
\hline Klassifikation & Ursachen/Krankheitsbilder \\
\hline Primäre ITP & keine auslösende Ursache erkennbar \\
\hline Sekundäre ITP & $\begin{array}{l}\text { medikamenteninduziert } \\
\text { Autoimmunerkrankung (SLE, rheumatoide Arthritis, und andere) } \\
\text { Antiphospholipid-Syndrom } \\
\text { CVID und Ähnliche } \\
\text { Lymphom (CLL, Hodgkin, und andere) } \\
\text { Evans-Syndrom } \\
\text { autoimmun-lymphoproliferatives Syndrom } \\
\text { Infektionen (HIV, Hepatitis B, Hepatitis C, H. pylori) } \\
\text { nach Impfung (z.B. Masern-Röteln-Mumps 1:40 000) } \\
\text { andere }\end{array}$ \\
\hline $\begin{array}{l}\text { Thrombozytopenie immunologisch } \\
\text { vermittelt, aber nicht als ITP } \\
\text { bezeichnet }\end{array}$ & $\begin{array}{l}\text { Thrombozytopenie bei GPIIb/IIIa-Inhibitor-Therapie } \\
\text { HIT } \\
\text { posttransfusionelle Purpura } \\
\text { schwangerschaftsassoziierte Thrombozytopenie } \\
\text { neonatale Allo-ITP }\end{array}$ \\
\hline $\begin{array}{l}\text { Weitere } \\
\text { Verbrauchsthrombozytopenien } \\
\text { (nicht immunologisch bedingt) }\end{array}$ & $\begin{array}{l}\text { mikroangiopathische hämolytische Anämien (TTP, HUS) } \\
\text { Verbrauchskoagulopathie } \\
\text { Von-Willebrand-Syndrom Typ IIb } \\
\text { massive Lungenembolie } \\
\text { Splenomegalie } \\
\text { große Hämangiome }\end{array}$ \\
\hline
\end{tabular}

SLE = Systemischer Lupus erythematodes, CVID = variables Immundefektsyndrom, CLL = chronische lymphatische Leukämie, HIV = humanes Immundefizienz-Virus, GPIIb/IIIa = Glykoprotein IIb/IIIa, HIT = heparininduzierte Thrombozytopenie, TTP = thrombotisch-thrombozytopenische Purpura, HUS = hämolytisch-urämisches Syndrom.
Was die Autoantikörperbildung initial auslöst, ist unbekannt. Studien finden Störungen der T- und B-regulatorischen Lymphozyten im Knochenmark (sogenannte Tregs, Bregs). Auch ein direkter toxischer Effekt von T-Lymphozyten auf Thrombozyten wird beschrieben [11-13].

\section{Relativer Thrombopoietinmangel}

Neben der oben genannten Immunreaktion gegen Thrombo- und Megakaryozyten wird als weiterer, die Thrombozytopoese hemmender Faktor eine nicht ausreichende Erhöhung der Thrombopoietinspiegel beschrieben. Die Spiegel sind zwar oft höher als bei gesunden Menschen, aber nicht so hoch wie bei anderen Erkrankungen, die einen vergleichbaren Thrombozytenmangel aufweisen (z.B. aplastische Anämie oder nach Chemotherapie). Man spricht deshalb von einem relativen Thrombopoietinmangel [14].

\section{Klinisches Bild}

Typische Blutungssymptome bei ITP:

- Petechien meist an den Beinen und der Mundschleimhaut, weniger häufig an Rumpf, Armen oder im Gesicht

- Blutungen der Schleimhäute von Mund und Nase

- urogenitale Blutungen, verstärkte Menstruationsblutungen

- verstärkte Blutungen bei geringen Verletzungen
- Hämatomneigung schon bei kleinen Traumen

- selten innere Blutungen (z.B. intrazerebrale Blutung) [15]

Als Folge verstärkten Blutverlustes kann sich eine Eisenmangelanämie entwickeln. Nicht typisch für die ITP sind flächenhafte Hämatome und Gelenkblutungen; diese findet man eher bei plasmatischen Gerinnungsstörungen. Fast ein Drittel aller Patienten mit chronischer ITP hat keine Symptome.

\section{Schweregrad, Stadieneinteilung und Verlauf}

\section{Schweregrad}

Die Behandlung der ITP sollte sich primär an der klinischen Blutungsneigung und nachrangig an der Thrombozytenzahl orientieren.

Zur Einschätzung der Blutungsschwere bei ITP wurden in der Vergangenheit zahlreiche Scores entwickelt (BoltonMaggs Score, Buchanan Score, Khellaf Score, Page Score), die sich jedoch - nicht zuletzt aufgrund ihrer Komplexität in der klinischen Praxis bisher nicht durchsetzen konnten. Deshalb wird weiterhin eine Orientierung an den Blutungsgraden der Weltgesundheitsorganisation (WHO) oder den Common Terminology Criteria for Adverse Events (CTCAE) des National Cancer Institute (NCI) empfohlen (Tab. 2). Sie sind in der klinischen Praxis etabliert und den meisten Ärzten geläufig $[16,17]$. 
Tab. 2. Blutungsgrade entsprechend WHO und NCI-CTCAE Version 4,0 $[16,17]$

\begin{tabular}{|c|c|}
\hline WHO Blutungsgrad & Definition \\
\hline 0 & keine Blutungszeichen \\
\hline I & $\begin{array}{l}\text { Petechien } \\
\text { kleine Hämatome, Ekchymosen }(<10 \mathrm{~cm}) \\
\text { Schleimhautblutungen (Mund, Nase) } \\
\text { Epistaxis (<1 h Dauer, keine ärztliche Intervention notwendig) } \\
\text { subkonjunktivale Blutungen } \\
\text { vaginale Blutungen (unabhängig von Menstruation, nicht mehr als } 2 \text { Binden/Tag notwendig) }\end{array}$ \\
\hline II (nicht transfusionspflichtig) & $\begin{array}{l}\text { Hämatome, Ekchymosen }(>10 \mathrm{~cm}) \\
\text { Epistaxis (>1 h Dauer oder Tamponade notwendig) } \\
\text { retinale Blutungen ohne Visusverminderung } \\
\text { vaginale Blutungen (unabhängig von Menstruation, mehr als } 2 \text { Binden/Tag notwendig) } \\
\text { Melaena, Hämatemesis, Hämoptysen, Hämaturie, Hämatochezie } \\
\text { Blutungen aus Punktionsstellen } \\
\text { Blutungen in Muskel und Gelenke }\end{array}$ \\
\hline III (transfusionspflichtig) & $\begin{array}{l}\text { Epistaxis } \\
\text { Schleimhautblutungen (Mund, Nase) } \\
\text { vaginale Blutungen } \\
\text { Melaena, Hämatemesis, Hämoptysen, Hämaturie, Hämatochezie } \\
\text { Blutungen aus Punktionsstellen } \\
\text { Blutungen in Muskel und Gelenke }\end{array}$ \\
\hline IV & $\begin{array}{l}\text { retinale Blutungen mit Visusverminderung } \\
\text { ZNS-Blutungen } \\
\text { andere Organblutungen, die die Funktion der betroffenen Organe (Gelenke, Muskulatur, } \\
\text { Niere, Lunge usw.) gefährden } \\
\text { letale Blutungen (in den NCI-CTCAE als Blutungsgrad V bezeichnet) }\end{array}$ \\
\hline
\end{tabular}

Kürzlich wurde von einer internationalen Arbeitsgruppe der SMOG Score vorgeschlagen, der für Blutungen der Haut (Skin), Schleimhäute (Mucosae) und Organe $(\mathrm{O})$ verschiedene Blutungsschweregrade $(G)$ genau definiert [18]. Der große Fortschritt des SMOG Scores ist, dass er die unterschiedlichen Blutungstypen und Lokalisationen besser als bisherige Scores erfasst. Dies ist gerade für die Beurteilung der Wirksamkeit neuer Therapien im Rahmen von Studien von höchstem Wert. Der SMOG Score wurde jedoch bisher nicht bezüglich seiner prognostischen Relevanz für klinische Therapieentscheidungen validiert. Ein weiterer Nachteil ist der hohe Zeitaufwand.

\section{Stadieneinteilung und Verlauf}

Traditionell spricht man von einer akuten ITP, wenn die Blutungsneigung anamnestisch erst vor kurzer Zeit aufgetreten ist, während die chronische ITP schon länger besteht, meist keine oder nur wenige Blutungssymptome hervorruft und manchmal nur zufällig bei einer Blutuntersuchung auffällt.

Die Begriffe «akut» und «chronisch» waren in der pädiatrischen und Erwachsenen-Hämatologie zum Teil unterschiedlich definiert. Deshalb und weil sich Therapie und Therapieziele mit der Krankheitsdauer und Schwere ändern, wurde die Zweiteilung verlassen, eine neue Einteilung in 3 Krankheitsund Therapiephasen [7] entwickelt und von den Leitlinien übernommen (Tab. 3).
Heute sind die Schweregrade und Stadien in der Kinderund Jugendmedizin genauso definiert wie bei den Erwachsenen [6].

\section{Prognose und Risiko}

Bei der ITP des Erwachsenen hat man einen langfristigen, chronischen Verlauf über mehrere Jahre oder sogar lebenslang postuliert. Kürzlich wurde jedoch gezeigt, dass doch ein bis zwei Drittel der chronischen ITP-Patienten ohne Splenektomie auch nach einem Jahr eine partielle oder komplette Remission erreichen [19, 20]. Die Remissionsrate bei plötzlich aufgetretener ITP mit Blutungsneigung war höher als bei einem schleichenden Beginn [19]. Dies ist wichtig für die Entscheidung, ob eine spontane Remission weiter abgewartet oder die Splenektomie angeboten werden sollte. In der Regel wird man nach 12 Monaten eine Splenektomie anbieten (siehe Abschnitt «Splenektomie als Zweitlinientherapie») [21].

Das Risiko tödlicher Blutungen steigt besonders nach dem 60. Lebensjahr [22, 23]. Neben Blutungen sind Infektionen durch die immunsuppressive Therapie eine ebenso wichtige Todesursache [24, 25]. Dies würde erklären, warum thrombozytopene Patienten, die unter chronischer Therapie stehen, eine höhere Mortalität haben als thrombozytopene Patienten 
Tab. 3. Stadieneinteilung bei ITP [7]

\begin{tabular}{lll}
\hline Stadium & Definition & Therapieziel \\
\hline Neu diagnostiziert & bis zu 3 Monate nach Diagnosestellung & $\begin{array}{l}\text { Blutungsstillung und Kuration, bei kurzer Therapiedauer unter Inkaufnahme } \\
\text { von Nebenwirkungen } \\
\text { Persistierend }\end{array}$ \\
$\begin{array}{l}\text { zwischen 3 und 12 Monaten nach } \\
\text { Diagnosestellung } \\
\text { mehr als 12 Monate nach Diagnosestellung, } \\
\text { eine spontane Remission ist jetzt nicht mehr } \\
\text { sehr wahrscheinlich }\end{array}$ & $\begin{array}{l}\text { Blutungstillung, Kuration; da Therapie häufiger längerfristig, sind Nutzen } \\
\text { und Nebenwirkungen stärker gegeneinander abzuwägen } \\
\text { Thrombozytopenie; Therapie nur bei schwereren Blutungen zwingend, bei } \\
\text { oligo- oder asymptomatischen Patienten auch Watch-and-Wait möglich }\end{array}$ \\
\hline
\end{tabular}

Tab. 4. Basisdiagnostik bei Erstvorstellung und zunächst nur klinischem Verdacht auf ITP

\begin{tabular}{ll}
\hline Anamnese & $\begin{array}{l}\text { aktuelle und frühere Blutungen, Infektionen, Medikamente, Alkohol, Schwangerschaft, frühere Thrombosen, } \\
\text { Familienanamnese, Berufsanamnese }\end{array}$ \\
Körperliche Untersuchung & Blutungszeichen, Lymphknoten, Lebergröße, Milzgröße usw. \\
Blutbild & EDTA und Citrat, zum Ausschluss einer Pseudothrombozytopenie \\
Blutausstrich & Begutachtung durch einen in der Diagnostik von hämatologischen Erkrankungen erfahrenen Arzt \\
Gerinnungsparameter & Thromboplastinzeit (Quick-Wert), aPTT \\
Blutgruppentestung & bei hohem Blutungsrisiko und für Eintrag in Notfallpass \\
Weiteres & Untersuchung auf irreguläre erythrozytäre Autoantikörper, insbesondere bei gleichzeitiger Anämie, Haemoccult, \\
& Urinuntersuchung auf Blut; Blutzucker/Urinzucker zum Ausschluss eines subklinischen Diabetes mellitus \\
Knochenmarkdiagnostik & Ausschluss von anderen hämatologischen Erkrankungen (Tab. 5); bei atypischen Befunden in der Basisdiagnostik \\
& empfohlen, insbesondere zu diskutieren bei älteren Patienten (>60 Jahre)
\end{tabular}

EDTA $=$ Ethylendiamintetraacetat, aPTT $=$ aktivierte partielle Thromboplastinzeit.

ohne Therapie [26]. Eine langfristige Immunsuppression sollte deshalb wenn irgend möglich vermieden werden.

Im Kindes- und Jugendalter sind schwere Blutungen eine Rarität. Die Blutungsneigung korreliert nicht mit der Thrombozytenzahl. Eine Splenektomie ist deshalb im Kindes- und Jugendalter als Ultima Ratio anzusehen (siehe Abschnitt «Splenektomie als Zweitlinientherapie»).

\section{Diagnostik}

\section{Basisdiagnostik bei klinischem Verdacht auf ITP}

Bei Erstvorstellung eines thrombozytopenen Patienten mit Verdacht auf ITP wird folgende Basisdiagnostik empfohlen (Tab. 4) (Empfehlungsgrad A):

Differenzialdiagnosen der ITP sind in Tabelle 5 zusammengefasst.

Eine ITP sollte nur diagnostiziert werden, wenn die Thrombozytenzahl $<100 \times 10^{9} / 1$ liegt (Empfehlungsgrad B) [7]. Die Zahl ist niedriger als der untere Grenzwert des Normbereichs (meist $150 \times 10^{9} / 1$ ), weil auch bei Gesunden Werte zwischen $100 \times 10^{9}$ und $150 \times 10^{9} / 1$ vorkommen können. Außerdem würde man eine Thrombozytopenie zwischen $100 \times 10^{9}$ und $150 \times 10^{9} / 1$ in der Regel nicht behandeln. Eine weitere Diagnostik wäre somit ohne therapeutische Konsequenz und sollte deshalb bei Werten in diesem Bereich unterbleiben.
Bei unauffälliger Anamnese ist der Nachweis einer isolierten Thrombozytopenie $<100 \times 10^{9} / 1$ bei normalen übrigen hämatologischen Parametern (Erythrozytenzahl, Leukozytenzahl, Differenzialblutbild, Gerinnungswerte) ausreichend für die Diagnose ITP. Zur weiteren Basisdiagnostik bei Erstvorstellung und zunächst nur klinischem Verdacht auf eine ITP siehe Tabelle 4. Eine Knochenmarkpunktion ist bei typischen Befunden zunächst nicht notwendig.

Zur Erstdiagnostik der ITP gehört bei Erwachsenen wie bei Kindern und Jugendlichen zwingend die Begutachtung des Blutausstriches durch einen in der Diagnostik von hämatologischen Erkrankungen erfahrenen Arzt. Eine thrombotisch-thrombozytopenische Purpura ist eine hämatologische Notfalldiagnose und darf nicht übersehen werden!

\section{Ausschluss einer medikamenteninduzierten \\ Thrombozytopenie}

Diese ist eine der wichtigsten Differenzialdiagnosen der ITP und kann manchmal nur durch wiederholte (!) Anamnese ausgeschlossen werden. Dabei müssen auch naturheilkundliche und nicht rezeptflichtige Medikamente erfragt werden, die von Laien häufig nicht als Medikamente im medizinischen Sinne gewertet und bei einer Anamnese nicht genannt werden. Die Inzidenz wird mit 0,1 pro 10000 pro Jahr angegeben (zum Vergleich ITP 0,2-0,4 pro 10000 pro Jahr, s.o.) [27]. Ein Medikament kann als Auslöser der Thrombozytopenie betrachtet werden, wenn folgende Kriterien erfüllt sind (Empfehlungsgrad B): 
Tab. 5. Differenzialdiagnose bei Verdacht auf ITP

Differenzialdiagnose
Pseudothrombozytopenie (EDTA-Thrombozytope
Hereditäre Thrombozytopenie
Medikamenteninduzierte Thrombozytopenie
Zytostatikainduzierte Thrombozytopenie
Virustatikainduzierte Thrombozytopenie
Heparininduzierte Thrombozytopenie
Posttransfusionelle Purpura
Schwangerschaftsassoziierte Thrombozytopenie
Lymphome

Lymphome

Infektionen (Viren, Bakterien, Parasiten)

\section{Lebererkrankungen}

\section{Alkoholabusus}

Sarkoidose

Schwere Vitaminmängel (B12, Folsäure, auch schwerster Eisenmangel)

Andere Autoimmunerkrankungen

\section{Evans-Syndrom}

Hämatologische Systemerkrankungen (akute Leukämie, myeloproliferative Syndrome, Myelodysplasie, Plasmozytom, Lymphome, CVID, autoimmun-lymphoproliferatives Syndrom, aplastische Anämie, paroxysmale nächtliche Hämoglobinurie, Graft-versus-Host-Erkrankung)

Thrombotisch-thrombozytopenische Purpura und hämolytischurämisches Syndrom

Von-Willebrand-Syndrom Typ IIb

Verbrauchskoagulopathie

Große Hämangiome (z.B. Kasabach-Merritt-Syndrom)
Anamnese, typische Befunde

1-5\% aller Blutproben $\rightarrow$ Bestimmung der Thrombozytenzahl in Citrat- oder Heparin-Blut

Familienanamnese, Untersuchung des Blutausstriches und MPV, ob sehr große (Makrothrombozytopenie) oder kleine Thrombozyten (WiskottAldrich-Syndrom) usw.

Anamnese, Testung auf medikamentenabhängige Thrombozyten-Antikörper Anamnese

Anamnese

Anamnese und HIT-Labordiagnostik

kurz zurückliegende Bluttransfusionen

nur schwangere Patientinnen, meist über $80 \times 10^{9}$ Thrombozyten/1

Anamnese, B-Symptome, Sonographie der Lymphknotenstationen, Milzgröße, eventuell Knochenmarkpunktion

Serologie auf HIV, CMV, EBV, Röteln, Parvovirus B19, Hantaviren; unter anderem bakteriologische Untersuchungen, Blutkultur bei Verdacht auf Sepsis, Blutausstrich bei Verdacht auf Malaria

Leberwerte, Hepatitis-Serologie, Sonographie ob Splenomegalie, Gaucher-Diagnostik

muss häufig gezielt erfragt werden

pneumologische Diagnostik

Labordiagnostik

Labordiagnostik für Lupus erythematodes, rheumatoide Arthritis, Antiphospholipid-Syndrom, Autoimmun-Thyreoiditis und andere Autoimmunsyndrome

Anämie, positiver direkter Antiglobulintest

Veränderung auch anderer Blutzellreihen und/oder der SerumImmunglobuline, Knochenmarkpunktion eventuell mit Durchflusszytometrie und Zytogenetik

meist weitere Symptome: Fieber, Hämolyse, Niereninsuffizienz, neurologische Symptome usw.

Von-Willebrand-Faktor-Analyse inklusive Multimeranalyse Veränderung weiterer Gerinnungsparameter klinisches Bild

EDTA $=$ Ethylendiamintetraacetat, MPV = mittleres Plättchenvolumen, HIT $=$ heparininduzierte Thrombozytopenie, HIV $=$ humanes Immundefizienz-Virus, CMV = Cytomegalovirus, EBV = Epstein-Barr-Virus, CVID = variables Immundefektsyndrom.

- Das Medikament wurde eingenommen, bevor die Thrombozytopenie auftrat, und nach Absetzen des Medikamentes normalisierte sich ihre Zahl wieder.

- Das Medikament wurde zusammen mit anderen Medikamenten eingenommen, als die Thrombozytopenie auftrat. Die Thrombozytopenie erholte sich nach Absetzen des Medikamentes, obgleich die anderen Medikamente weitergeführt wurden.

- Andere Ursachen einer Thrombozytopenie wurden ausgeschlossen.

- Eine wiederholte Exposition führt zur erneuten Thrombozytopenie.

Obwohl die ersten drei Kriterien die Diagnose nicht $100 \%$ ig sichern, wird man nur in Ausnahmefällen, z.B. bei lebenswichtigen Medikamenten, einen Re-Expositionsversuch durchführen und damit die Diagnose definitiv sichern.

Häufige Auslöser sind: Chinin, Chinidin, Trimethoprim/ Sulfamethoxazol, Vancomycin, Penicilline, Rifampizin, Carbamazepin, Ceftriaxon, Ibuprofen, Mirtazapin, Oxaliplatin, Suramin, Abciximab, Tirofiban, Eptifibatide und Heparine [28]. Der Verdacht auf eine medikamenteninduzierte Thrombozytopenie kann durch den immunologischen Nachweis medikamentenabhängiger antithrombozytärer Antikörper bestätigt werden. Dieses In-vitro-Verfahren ist jedoch nur in Speziallaboratorien verfügbar und in seiner Aussagekraft beschränkt. Wenn die Immunreaktion durch spezielle Metabolite vom Individuum ausgelöst wurde, versagt es [28, 29]. 
Tab. 6. Weiterführende Diagnostik

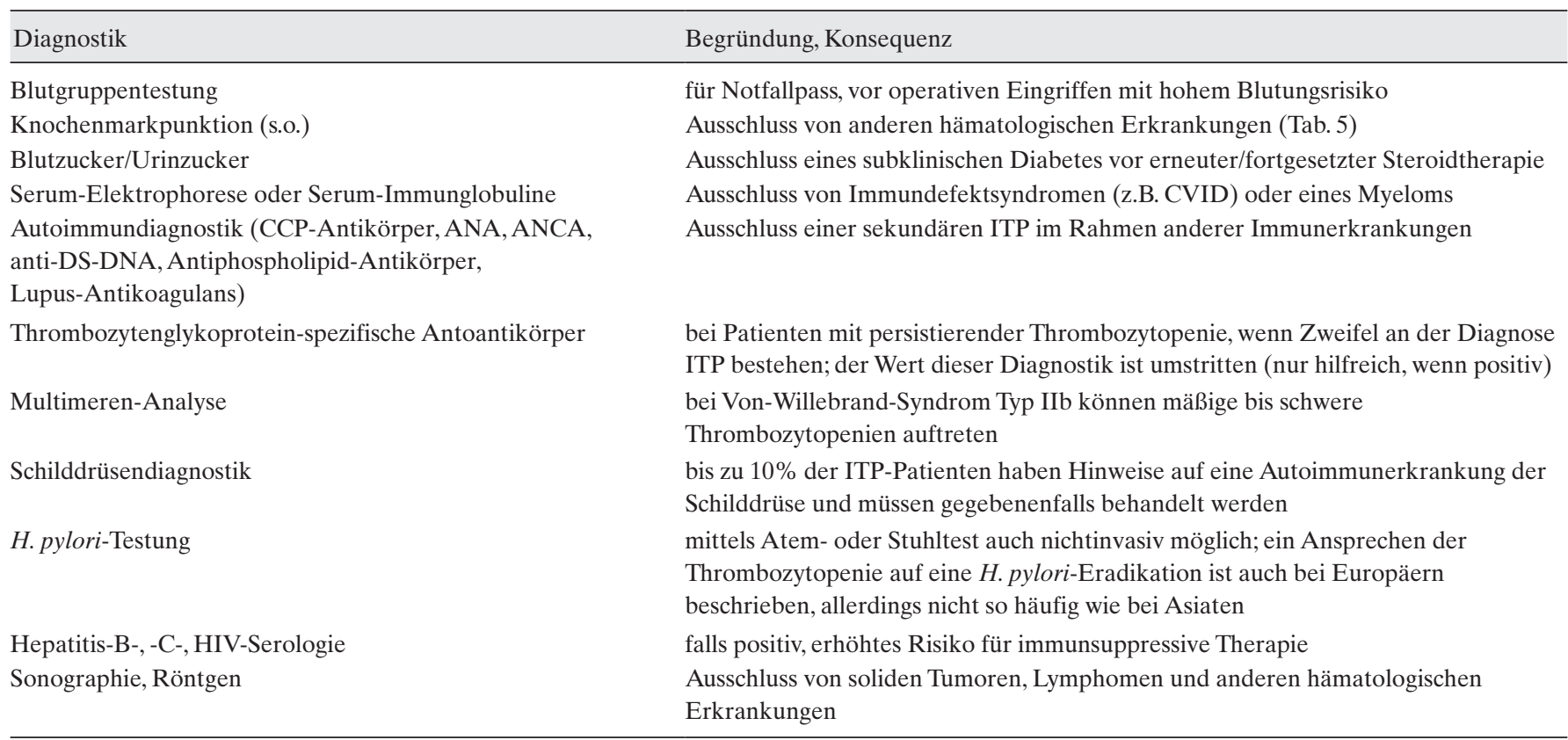

CVID = Variables Immundefektsyndrom, CCP = zyklische citrullinierte Peptide, ANA = antinukleäre Antikörper, ANCA = anti-Neutrophile cytoplasmatische Antikörper, DS-DNA = doppelsträngige DNA.

In der Regel hat die medikamenteninduzierte ITP einen akuten Verlauf. Nach Absetzen des Medikamentes erholt sich die Thrombozytenzahl rasch wieder. Nur für ganz wenige Medikamente wurde beschrieben, dass sie eine chronische ITP auslösen können, die auch nach Absetzen des Medikamentes persistiert: Trimethoprim/Sulfamethoxazol, Procainamid, Chinidin, die in der Rheumatherapie heute seltener eingesetzten Goldverbindungen und einige wenige andere [29, 30].

Eine aktuelle Liste von Arzneistoffen, für die medikamenteninduzierte Thrombozytopenien beschrieben wurden, findet sich unter www.ouhsc.edu/platelets/ditp.html.

\section{Knochenmarkpunktion}

Eine Knochenmarkpunktion ist bei typischen Befunden nicht notwendig (Empfehlungsgrad B). Eine Knochenmarkdiagnostik sollte aber immer erwogen werden,

- wenn neben der Thrombozytenzahl auch andere Laborwerte, insbesondere Leukozyten- und Erythrozytenparameter verändert sind,

- wenn atypische anamnestische oder körperliche Untersuchungsbefunde vorliegen (z.B. vergrößerte Lymphknoten, Hepatosplenomegalie),

- bei Patienten > 60 Jahre, wegen der zunehmenden Häufigkeit alternativer Diagnosen: Lymphome, myelodysplastische Syndrome, Plasmozytom und andere (in einer Studie ergaben sich bei bis zu $10 \%$ der Patienten im weiteren Krankheitsverlauf Änderung der ITP-Diagnose) [31],

- vor einer Splenektomie, um alternative Diagnosen vor diesem irreversiblen Eingriff mit größerer Sicherheit auszuschließen. Bei Patienten ohne atypische Symptome und mit charakteristischem Verlauf, z.B. gutem Ansprechen auf eine vorherige Steroidtherapie, kann auf die Knochenmarkpunktion verzichtet werden.

\section{Weiterführende Diagnostik bei persistierender oder chronischer} ITP

Eine weiterführende Diagnostik ist sinnvoll (Empfehlungs$\operatorname{grad} B)$ :

- bei Patienten, deren Thrombozytenwerte unter der Erstlinientherapie (zumeist Steroide) gar nicht oder nur minimal angestiegen sind (primäres Therapieversagen),

- bei Patienten, deren Thrombozytenzahl auf die Erstlinientherapie zunächst angesprochen hat, die dann aber bei der notwendigen Dosisreduktion des Steroids oder im weiteren Krankheitsverlauf einen erneuten Thrombozytenabfall entwickelt haben (sekundäres Therapieversagen).

Diagnostische Maßnahmen und ihre Begründung sind in Tabelle 6 aufgeführt.

\section{Helicobacter pylori}

Eine Helicobacter pylori-Infektion kann in Einzelfällen das Auftreten oder Persistieren einer ITP begünstigen. Die Eradikationstherapie ist bei asiatischen Patienten relativ erfolgreicher [32], angesichts der Einfachheit der Diagnostik und der niedrigen Kosten aber auch bei Europäern sinnvoll (Empfehlungsgrad B).

\section{Thrombozyten-Antikörper}

Die Bestimmung von Thrombozyten-Antikörpern kann bisher nicht als Routineuntersuchung empfohlen werden 


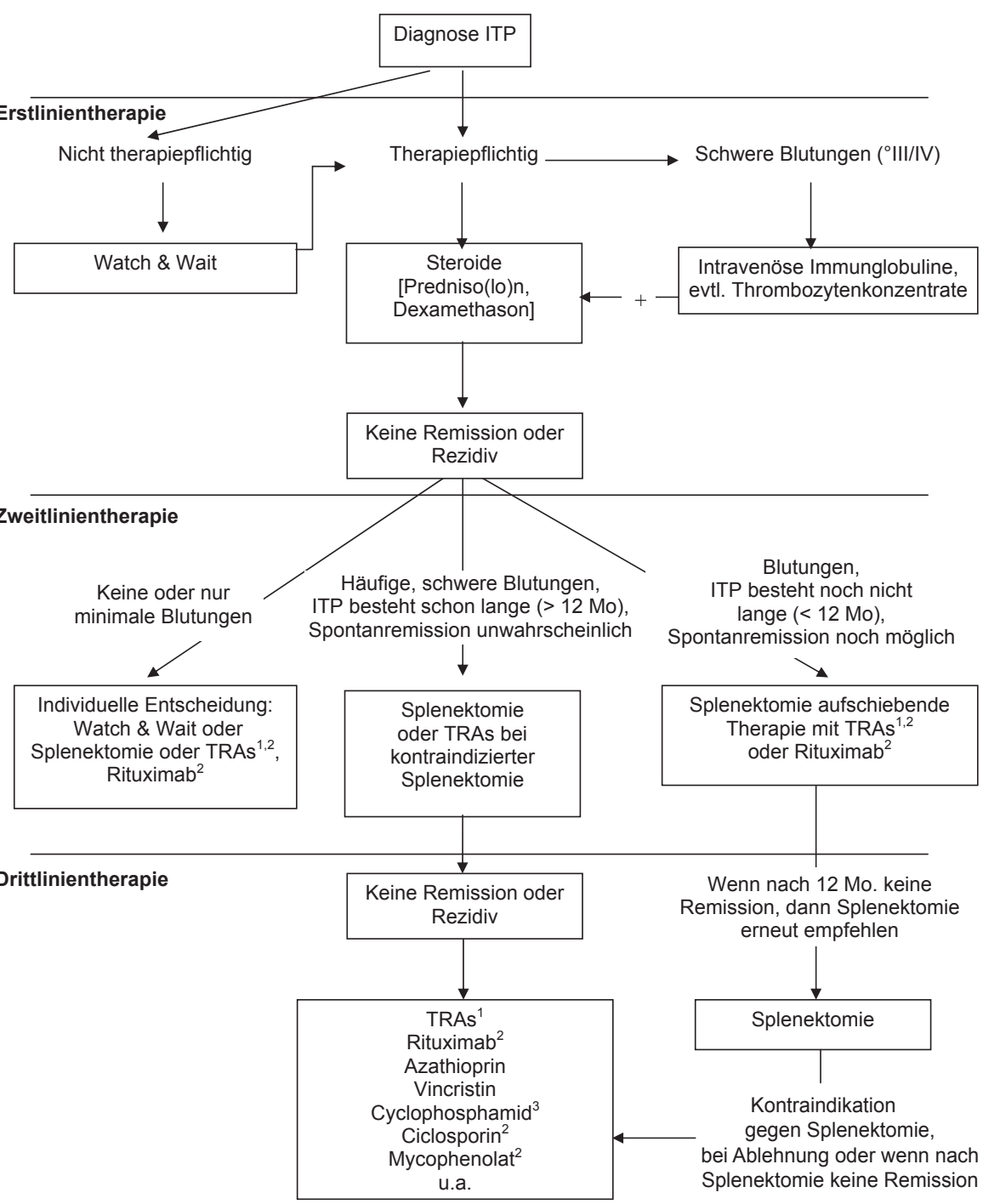

Abb. 1. Algorithmus zu Therapieempfehlungen bei Immunthrombozytopenie. TRAs = Thrombopoietinrezeptor-Agonisten: Angaben nach Zulassungsbehörden. ${ }^{1} \mathrm{Im}$ Vergleich zu Rituximab, Azathioprin, und anderen Zweitund Drittlinientherapien haben TRAs die bessere Evidenzstärke (Stärke Ib) und den höheren Empfehlungsgrad (B). ${ }^{2}$ TRAs haben in Deutschland und Österreich keine arzneimittelrechtliche Zulassung vor Splenektomie (es sei denn, die Splenektomie ist medizinisch kontraindiziert). In der Schweiz sind TRAs vor Splenektomie nicht off-label. Für Zulassungstext Schweiz siehe www.swissmedicinfo.ch. Rituximab ist in keinem Land zur Behandlung der ITP arzneimittelrechtlich zugelassen. ${ }^{3}$ Cyclophosphamid ist in Deutschland nur für bedrohlich verlaufende Immunerkrankungen zugelassen.

(Empfehlungsgrad B). Bei Patienten mit persistierender Thrombozytopenie und atypischen Befunden kann der Nachweis von Thrombozyten-Antikörpern in Einzelfällen dazu beitragen, die Diagnose ITP zu sichern bzw. andere Differenzialdiagnosen auszuschließen.

Hilfreich ist nur der positive Nachweis von Antikörpern. Wenn Thrombozyten-Antikörper nicht nachgewiesen werden können, schließt dies die Diagnose ITP nicht aus. Glykoprotein-spezifische Tests (MAIPA: monoklonale Antikörper-spezifische Immobilisierung von Plättchenantigenen) sollten den alten unspezifischen Tests (z.B. Immunfluoreszenztest auf Thrombozyten-assoziierte Immunglobuline) vorgezogen werden. Da aber auch die neuen Testverfahren nicht standardisiert und falsch-positive und falsch-negative Befunde nicht selten sind [33], wird empfohlen, zur besseren differenzialdiagnostischen Bewertung die Sensitivitäts- und Spezifitätszahlen des verwendeten Tests beim jeweiligen Labor zu erfragen.

Davon muss die Untersuchung auf medikamentenabhängige Thrombozyten-Antikörper bei medikamenteninduzierter Thrombozytopenie unterschieden werden (s.o.).

\section{Therapie, Allgemeines}

Ein Algorithmus zur Therapiesequenz bei Patienten mit ITP ist in Abbildung 1 dargestellt. Er stellt einen Expertenvorschlag zur Strukturierung des therapeutischen Vorgehens dar. Weitere Informationen zur Wirksamkeit der verschiedenen Therapieoptionen und zum Zulassungsstatus der Arzneimittel finden sich in den nachfolgenden Abschnitten.

Arzneimittel und Dosierungen der medikamentösen Therapie sind in Tabelle 7 aufgeführt. Informationen zum Zulassungsstatus finden sich in Tabelle 8 .

\section{Management von ITP-Patienten, Erstlinientherapie der neu diagnostizierten ITP}

Die Indikation zur Erstlinientherapie orientiert sich primär an der Blutungsneigung und nur nachrangig an der Thrombozytenzahl. 
Tab. 7. Häufig eingesetzte Medikamente und Dosierungen bei IT

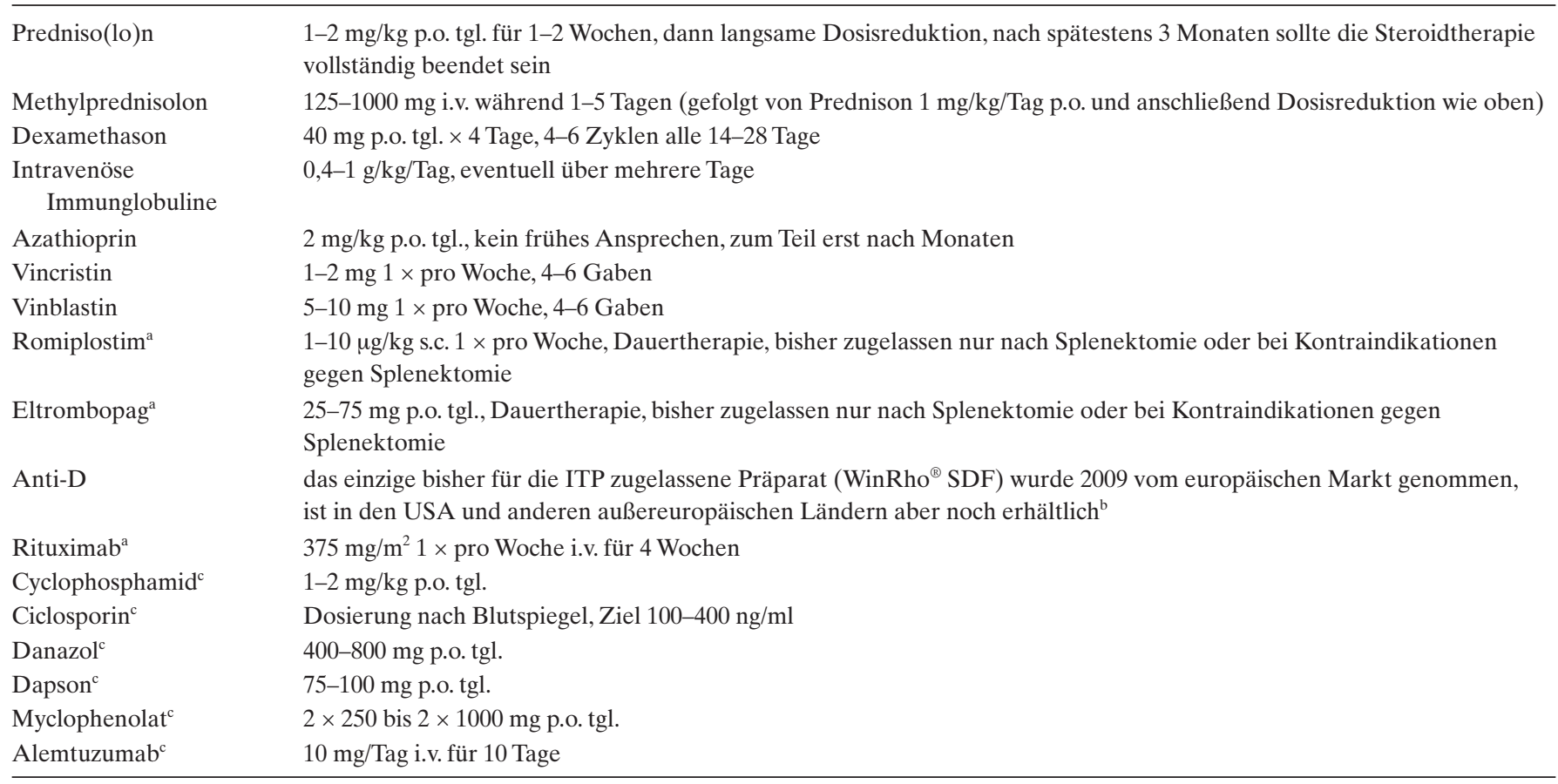

a TRAs haben in Deutschland und Österreich keine arzneimittelrechtliche Zulassung vor Splenektomie (es sei denn, die Splenektomie ist medizinisch kontraindiziert). In der Schweiz sind TRAs vor Splenektomie nicht off-label. Für Zulassung Schweiz, siehe Tabelle 11 und www.swissmedicinfo.ch.

${ }^{\mathrm{b}}$ Andere Anti-D-Immunglobuline (z.B. Rhophylac ${ }^{\circledR}$, Rhesonativ ${ }^{\circledR}$ ) sind nicht für die ITP, sondern nur zur Prophylaxe des Morbus haemolyticus neonatorum zugelassen.

${ }^{\mathrm{c}}$ Keine arzneimittelrechtliche Zulassung zur Behandlung der ITP. Bei nicht zugelassenen Therapien sollte vorher eine ausführliche Patientenaufklärung erfolgen und in der Krankenakte dokumentiert werden.

p.o. $=$ Oral $($ per os $)$, i.v. $=$ intravenös, s.c. $=$ subkutan, tgl. $=$ täglich .

\section{Schwere Blutungen}

Bei allen Patienten mit ITP und WHO Grad-III- oder Grad-IV-Blutungen (Tab. 2) besteht eine Therapieindikation unabhängig von der Thrombozytenzahl (Empfehlungsgrad A). Eine stationäre Aufnahme wird empfohlen.

\section{Mittelschwere Blutungen}

Bei neu diagnostizierten Patienten mit mittelschweren Blutungen (WHO Grad II) wird man sich in der Regel ebenfalls $\mathrm{zu}$ einer Erstlinientherapie entschließen (Empfehlungsgrad $B)$. Eine «Watch-and-Wait»-Strategie ist in begründeten Einzelfällen (schwer einstellbarer Diabetes, Ablehnung von Kortikosteroiden, mangelnde Compliance, Wunsch des Patienten) aber auch vertretbar. Die Indikation zur stationären Aufnahme sollte großzügig gestellt werden.

\section{Keine oder nur leichte Blutungen}

Bei neu diagnostizierten Patienten mit geringer oder gänzlich fehlender Blutungsneigung (WHO Grad 0 und I) wird man meist auch eine Erstlinientherapie anbieten, schon allein weil der Patient beunruhigt und ein Therapieverzicht kaum zu vermitteln ist (Empfehlungsgrad B). Außerdem ist beim neu diagnostizierten Patienten der weitere Krankheitsverlauf nicht absehbar, d.h. ob die Blutungsneigung «blande» bleibt oder sich verschlimmern wird. Allerdings ist die Therapienotwendigkeit bei oligo- oder asymptomatischen Patienten, insbesondere bei jungen Patienten mit niedrigen Thrombozytenzahlen, nicht gesichert. Nutzen und Risiken (z.B. berufliches Verletzungsrisiko, Komorbiditäten) müssen individuell abgewogen werden. Das Register der Intercontinental Cooperative Immune Thrombocytopenia Study Group zeigt aber auch, dass fast 30\% der Erwachsenen mit neu diagnostizierter ITP zunächst mit einer Watch-and-Wait-Strategie erfolgreich betreut wurden [34].

Es ist zu überlegen, ob man beim neu diagnostizierten, aber asymptomatischen ITP-Patienten einen Therapieversuch anbietet, um zu sehen, ob Steroide oder intravenös verabreichte Immunglobuline im Blutungs-/Operationsfall ausreichend und schnell genug wirksam sind (Empfehlungsgrad 0). Eine stationäre Aufnahme ist bei fehlender Blutungsneigung in der Regel nicht notwendig.

In der Kinder- und Jugendmedizin soll die Therapieindikation ausschließlich durch klinische Symptome ausgelöst werden. Die Thrombozytenzahlen sind kein Entscheidungskriterium bei der neu diagnostizierten ITP. Individuelle Gesichtspunkte wie Alter, Verletzungsanfälligkeit oder psychosoziale Aspekte können eine Rolle spielen.

Bei der neu diagnostizierten ITP im Kindes- und Jugendalter ist eine Therapie in den allermeisten Fällen verzichtbar. 
Tab. 8. Zulassungsstatus von Medikamenten in Deutschland, Österreich und der Schweiz

\begin{tabular}{|c|c|c|}
\hline Substanz & Zulassung & Anmerkungen \\
\hline Alemtuzumab & nein & Fallberichte; Alemtuzumab ist in Europa zurzeit als Arzneimittel nicht zugelassen \\
\hline Anti-D-Immunglobulin & nein & $\begin{array}{l}\text { das einzige bisher für die ITP zugelassene Präparat (WinRho }{ }^{\circledR} \text { SDF) wurde } 2009 \text { in der EU vom Markt } \\
\text { genommen, ist in den USA und anderen außereuropäischen Ländern jedoch erhältlich; andere Anti-D- } \\
\text { Immunglobuline (z.B. Rhophylac }{ }^{\circledR}, \text { Rhesonativ }^{\circledR} \text { ) sind nicht für die Therapie der ITP zugelassen }\end{array}$ \\
\hline Azathioprin & ja & chronische, refraktäre ITP \\
\hline Ciclosporin & nein & Fallberichte \\
\hline Cyclophosphamid & ja & bedrohlich verlaufende Autoimmunerkrankungen \\
\hline Danazol & nein & $\begin{array}{l}\text { Fallberichte; in Deutschland ist kein Danazol mehr zugelassen; zugelassene Präparate gibt es unter } \\
\text { anderem in Österreich und in der Schweiz }\end{array}$ \\
\hline Dapson & nein & Fallberichte \\
\hline \multirow[t]{2}{*}{ Dexamethason } & ja & Deutschland, Österreich: Autoimmunerkrankungen \\
\hline & nein & Schweiz: Autoimmunerkrankungen wie rheumatoide Arthritis \\
\hline Immunglobuline & ja & $\begin{array}{l}\text { primäre ITP bei Patienten mit hohem Blutungsrisiko oder vor Operationen zur Korrektur der } \\
\text { Thrombozytenzahl }\end{array}$ \\
\hline Methylprednisolon & ja & Erkrankungen, die einer systemischen Therapie mit Glukokortikoiden bedürfen \\
\hline Mycophenolat & nein & Fallberichte \\
\hline Predniso(lo)n & ja & Erkrankungen aus der Hämatologie, die einer systemischen Behandlung mit Glukokortikoiden bedürfen \\
\hline Rituximab & nein & in Phase-II- und -III-Studien eingesetzt \\
\hline Romiplostim & ja & $\begin{array}{l}\text { erwachsene, splenektomierte Patienten, die gegen andere Therapien refraktär sind; Anwendung kann für } \\
\text { erwachsene, nicht splenektomierte Patienten, bei denen eine Operation kontraindiziert ist, als Zweitlinien- } \\
\text { therapie in Betracht gezogen werden; in der Schweiz ist die Gabe vor Splenektomie nicht off-label }\end{array}$ \\
\hline Vinblastin & nein & Fallberichte \\
\hline Vincristin & ja & ITP, nur nach Steroidtherapie und Splenektomie, keine Primärtherapie \\
\hline
\end{tabular}

Die arzneimittelrechtliche Zulassung in der Schweiz unterscheidet sich zum Teil von der EU-Zulassung für Deutschland und Österreich.

Für Zulassung Schweiz, siehe auch www.swissmedicinfo.ch.

Hämatome und Sugillationen stellen keine Therapieindikation dar. Schleimhautblutungen, die nicht mit lokalen Mitteln kontrollierbar sind, können jedoch zur Therapie führen. Bei neuen Patienten ist eine stationäre Überwachung mit initialer Bettruhe unter Aufsicht ebenfalls sinnvoll.

\section{Medikamente für die Erstlinientherapie}

Als Erstlinientherapie soll eine Behandlung mit Kortikosteroiden angeboten werden, außer es bestehen Kontraindikationen gegen Kortikosteroide (Empfehlungsgrad A). Die initial hohe Steroiddosis sollte nach 1-2 Wochen reduziert, dann ausgeschlichen werden. Eine maximale Therapiedauer von 2-3 Monaten sollte wegen des Risikos schwerer Steroidnebenwirkungen auf keinem Fall überschritten werden.

Die Wahl des Kortikosteroids, Predniso(lo)n oder Dexamethason, richtet sich nach der Expertise des Arztes. Ein Wirksamkeitsunterschied konnte bisher nicht gezeigt werden [35]. Mehrere Studien zu diesem Thema sind zurzeit aktiviert und werden diese Frage in naher Zukunft beantworten.

\section{Thrombozytenschwellenwerte}

Die ITP-Leitlinie der ASH von 1996 sah eine zwingende Therapieindikation bei niedrigen Thrombozytenwerten, auch wenn der Patient völlig beschwerdefrei war [36]. Dies hatte dazu geführt, dass viele Patienten über Monate bis Jahre mit Kortikosteroiden behandelt und zum Teil schwerste Nebenwirkungen (Cushing, Infektionen, Diabetes) in Kauf genommen wurden, nur um die Werte «hoch zu halten». Der Internationale Consensus-Report von 2009 [4] gab dann lediglich vor, dass bei Werten über $50 \times 10^{9} / 1$ eine Therapie kontraindiziert sei. Das Vorgehen bei niedrigeren Werten wurde nicht stringent vorgegeben. Die Experten der ASH-Leitlinie von 2010 empfehlen wieder einen Schwellenwert von $30 \times 10^{9} / 1$ allein aufgrund der Tatsache, dass dieser Wert allgemein akzeptiert sei und weil auch nichts gegen diesen Schwellenwert spreche (Evidenzlevel V, 5). Diese Empfehlung mag der haftungsrechtlichen Absicherung im angloamerikanischen Gesundheitssystem geschuldet sein.

Die Leitlinien der DGHO, ÖGHO, SGH + SSH und GPOH haben sich von der strikten Fixierung auf die Thrombozytenzahl gelöst $[1-3,6]$. Sicher steigt das Blutungs- und Mortalitätsrisiko, wenn eine Thrombozytenzahl von $30 \times 10^{9} / 1$ unterschritten wird, jedoch bestehen große individuelle Schwankungen. Man wird beim erstmaligen Auftreten der Krankheit und bei Thrombozytenwerten unter $20 \times 10^{9}-30 \times$ $10^{9} / 1$ in der Regel eine Behandlung anbieten. Je länger die 
Krankheit aber andauert, desto wichtiger wird die individuelle Blutungsneigung im Vergleich zur Thrombozytenzahl für den weiteren Entscheidungsprozess. Die Nebenwirkungen der Therapie müssen gegen den Nutzen abgewogen werden. Insbesondere in späteren Therapielinien wäre es somit kein Fehler, auch bei sehr niedrigen Werten eine Watch-and-Wait-Strategie zu verfolgen, solange der Patient nicht oder nur minimal blutet.

\section{Therapie von Notfällen}

\section{Notfalltherapie mit intravenösen Immunglobulinen}

Intravenös applizierte Immunglobuline führen $\mathrm{zu}$ einem raschen, oft kurzfristigen Thrombozytenanstieg für 2-4 Wochen, danach fallen die Werte aber in der Regel wieder auf das Ausgangsniveau. Eine dauerhafte Remission wird selten erreicht. Dadurch beschränkt sich ihre Indikation auf Situationen, in denen ein schneller Thrombozytenanstieg erreicht werden muss (Blutungen, nicht aufschiebbare Operationen) oder wenn höher dosierte Steroide vermieden werden sollen (z.B. Schwangerschaft).

Die intravenöse Gabe von Immunglobulinen ist bei ITPPatienten mit Grad-III- oder Grad-IV-Blutungen immer indiziert, unabhängig von der Thrombozytenzahl (Empfehlungsgrad A). Bei mittelschweren Blutungen (Grad II) werden sie meist ebenfalls angeboten. Bei leichten Blutungen ( Grad I) ist die Indikation für intravenöse Immunglobuline nur in Einzelfällen gegeben (alter Patient, schwere Thrombozytopenie usw.). Die Gabe von intravenösen Immunglobulinen ist bei ITP-Patienten ohne klinische Blutungsneigung auch bei sehr niedrigen Thrombozytenzahlen nicht indiziert, außer zur Vorbereitung nicht aufschiebbarer Operationen (Empfehlungsgrad B). Bezüglich Notfalltherapie mit Thrombozytenkonzentraten siehe unten.

Die Gabe von intravenösen Immunglobulinen sollte bei schweren Blutungen mit einer Kortikosteroidtherapie (z.B. Methylprednisolon $15 \mathrm{mg} / \mathrm{kg}$, max. $1000 \mathrm{mg}$ intravenös) kombiniert werden (Empfehlungsgrad $A$ ).

Die Notfalltherapie in der Kinder- und Jugendmedizin ist identisch.

\section{Notfalltherapie mit Anti-D-Immunglobulinen}

Als erstes und bisher einziges Anti-D-Immunglobulin zur Behandlung der ITP wurde in den 1990er-Jahren das Präparat WinRho $^{\mathrm{TM}}$ in den USA und später auch in Europa zugelassen. 2009 wurde WinRho ${ }^{\text {TM }}$ jedoch wegen seltener Hämolysen und Gerinnungsveränderungen in Europa wieder vom Markt genommen; es ist in den USA und einigen anderen Ländern aber noch erhältlich. Die Zulassung der in Deutschland, Österreich und der Schweiz erhältlichen Anti-D-Präparate (Rhophylac ${ }^{\circledR}$, Rhesonativ ${ }^{\circledR}$ und andere) ist auf die Prophylaxe des Morbus haemolyticus neonatorum beschränkt. Neue Anti-D-Präparate (Rozrolimupab) sind in der Entwicklung.

\section{Notfalltherapie mit Thrombozytenkonzentraten}

Bei schweren Blutungen (WHO Grad III, VI) kann mit Thrombozytenkonzentraten ein kurzfristiger Anstieg der Thrombozytenzahl und ein Sistieren der Blutung erreicht werden [37] (Empfehlungsgrad B). Gleichzeitig sollten immer Steroide und Immunglobuline verabreicht werden. Eine Stimulation der Bildung von Thrombozyten-Antikörpern durch die Thrombozytengabe wurde bisher nicht beobachtet.

\section{Zweitlinientherapie bei persistierender oder chronischer ITP}

Eine Zweitlinientherapie ist indiziert, wenn der Patient auf die Erstlinientherapie mit Steroiden nur partiell oder gar nicht anspricht oder wenn er nach initialer Therapieansprache einen erneuten Thrombozytenabfall entwickelt (Empfehlungsgrad B). Die Indikation zur Zweitlinientherapie orientiert sich dabei primär an der Blutungsneigung und weniger an der Thrombozytenzahl.

Bei allen Patienten mit persistierender oder chronischer ITP und WHO Grad-III- oder Grad-IV-Blutungen besteht eine klare Therapieindikation. Bei Patienten mit chronischer ITP ohne (WHO Grad 0) oder nur mit leichten Blutungen (WHO Grad I/II) besteht unabhängig von der Schwere der Thrombozytopenie keine zwingende Therapieindikation. Die Erfahrung zeigt jedoch, dass sich viele dieser asymptomatischen Patienten angesichts des als bedrohlich empfundenen Blutungsrisikos für eine Zweitlinientherapie entscheiden. Eine Watch-and-Wait-Strategie wäre dennoch und selbst bei sehr niedrigen Werten kein Fehler, solange der Patient nicht blutet.

\section{Splenektomie als Zweitlinientherapie}

Die Splenektomie ist die Zweitlinientherapie mit der höchsten Rate an dauerhaften Remissionen in dem Sinne, dass keine weitere Behandlung notwendig ist (zwei Drittel partielle oder komplette Remissionen). Thrombopoetinrezeptor-Agonisten (TRAs) erreichen zwar noch höhere Remissionsraten, wenn man nur das Ansprechen der Thrombozytenzahl betrachtet. Wenn man sie dann aber absetzt, fallen in der Regel die Thrombozytenzahlen wieder ab.

Es besteht eine klare Indikation zur Splenektomie für alle Patienten mit persistierender oder chronischer Thrombozytopenie und schweren Blutungen WHO Grad III, IV (Empfehlungsgrad $A$ ), die ein ungenügendes Ansprechen auf alle anderen, bisherigen Therapiemodalitäten aufweisen. Bei dringlicher Therapie (z.B. lebensbedrohliche Blutung, die nicht auf intravenös verabreichte Immunglobuline anspricht!) ist die Splenektomie die Therapie der Wahl, weil die alternative Gabe von TRAs oder Rituximab Zeit braucht (häufig $>1$ Woche).

Es besteht keine zwingende Indikation zur Splenektomie für Patienten mit chronischer ITP, deren Thrombozytenzahlen 
Tab. 9. Risiken und Komplikationen nach Splenektomie
Post-operative Morbidität etwa 10\% (Wundinfektionen, Pneumonien)

Post-operative Mortalität < 1\% [40] (bei älteren Patienten durchaus höher [44, 45])

OPSI (Sepsisrisiko 3-fach erhöht [46])

Postoperative Thrombozytose (bei $>10^{6}$, Acetylsalicylsäure oder NMH erwägen)

Venöse Thromboembolien [46]

Pulmonale Hypertonie

Erhöhtes Infektionsrisiko bei Aufenthalt in Gebieten mit Malaria und Babesiose

OPSI $=$ Overwhelming Post-Splenectomy Infection . bei $<30 \times 10^{9} / 1$ liegen und die keine, leichte oder mittelschwere Blutungen (WHO Grad 0, I, II) haben. Hier muss individuell entschieden werden (Empfehlungsgrad B).

Studien zeigen, dass in der klinischen Realität bis zu $10 \%$ der ITP-Diagnosen nicht korrekt sind und im weiteren Verlauf geändert werden müssen [31]. Deshalb wird vor der Splenektomie zur Diagnosesicherung eine weitere Abklärung inklusive Knochenmarkpunktion (Tab. 5) empfohlen. Dies gilt insbesondere bei atypischem Verlauf (z.B. völlig fehlendes Ansprechen auf Kortikosteroide und Immunglobuline) und/ oder Patienten $>60$ Jahre. Alle Patienten sollten präoperativ gegen Pneumokokken, Haemophilus influenzae B und Meningokokken geimpft werden (Empfehlungsgrad B) [38] (s.a. Onkopedia-Leitlinie «Prävention von Infektionen undThrombosen nach Splenektomie oder bei funktioneller Asplenie» (www.dgho-onkopedia.de)).

Anmerkung: Meningokokken-Konjugatimpfstoff sollte nur intramuskulär gegeben werden, was bei schwerer Thrombozytopenie ein Problem sein kann. Dann wären die älteren Meningokokken-Polysaccharidimpfstoffe, die man auch subkutan geben kann, vorzuziehen.

Ein Drittel der Patienten erleidet nach Splenektomie kurzfristig oder auch nach Jahren einen therapiewürdigen Rückfall [39-41]. Es ist bisher nicht möglich vorherzusagen, welcher Patient auf eine Splenektomie ansprechen wird und welcher nicht. Der Internationale Consensus-Report empfiehlt die Splenektomie, wenn der Abbau radioaktiv markierter Thrombozyten vorwiegend in der Milz erfolgt [4]. Diese Empfehlung kann jedoch aufgrund widersprüchlicher Daten bisher nicht als verbindlich betrachtet werden. Auch wenn die Szintigraphie zeigt, dass die Thrombozyten primär nicht in der Milz abgebaut werden, hat die Splenektomie immer noch eine relativ gute Erfolgsrate von 35-40\% [42, 43]. Die Szintigraphie ist immer sinnvoll, wenn es nach Splenektomie zu einem Rezidiv kommt und wenn eine Nebenmilz ausgeschlossen werden soll.

Da Spontanremissionen der ITP bis 12 Monate nach Diagnosestellung relativ häufig vorkommen [19] - danach sind sie eher selten - sollte versucht werden, die Splenektomie bis zu diesem Zeitpunkt hinauszuschieben. Gegebenenfalls kann eine sogenannte «Splenektomie aufschiebende» medikamentöse Therapie angeboten werden (Empfehlungsgrad 0) [21].

Risiken und Komplikation der Splenektomie sind in Tabelle 9 zusammengefasst.

\section{Splenektomie aufschiebende Therapien}

In der klinischen Realität werden nur etwa ein Drittel der Patienten mit chronischer ITP splenektomiert. Viele Patienten lehnen diesen Eingriff ab und fragen nach sogenannten Splenektomie aufschiebenden oder Splenektomie ersetzenden medikamentösen Therapien. Dies ist zum Teil damit begründet, dass in anderen Ländern medikamentöse Therapiealternativen (TRAs und Rituximab) vor der Splenektomie eingesetzt werden können. Aufgrund der bis zum Ablauf des ersten Erkrankungsjahres häufigen spontanen Remissionen ist das Hinausschieben der Operation aber auch medizinisch sinnvoll und wird von vielen Leitlinien empfohlen (Empfehlungsgrad B) [21]. Wenn es nach dieser Zeit jedoch nicht zu einer Remission gekommen ist und der Patient eine relevante Blutungsneigung hat, dann macht eine Splenektomie durchaus Sinn. Spontane Remissionen sind zwar auch noch später möglich, aber nicht so häufig, als dass man dies zur Grundlage von Therapieentscheidungen machen könnte.

Die TRAs Romiplostim und Eltrombopag sind in der Europäischen Union (EU) seit 2009 bzw. 2010 für die Behandlung splenektomierter Patienten mit chronischer ITP zugelassen. Romiplostim und Eltrombopag können als Zweitlinientherapie für nicht splenektomierte Patienten nur dann in Betracht gezogen werden, wenn eine Operation kontraindiziert ist. In der Schweiz sind TRAs vor Splenektomie nicht «off-label». Rituximab hat keine arzneimittelrechtliche Zulassung für die chronische ITP, wird aber dennoch häufig als Splenektomie aufschiebende Therapie eingesetzt (Empfehlungsgrad B) $[47,48]$.

Der Internationale Consensus-Report gibt TRAs eine höhere Empfehlung (Grad A) als der Splenektomie (Grad C) [4]. Die ASH-Leitlinie wertet dagegen die Splenektomie höher (Empfehlungsgrad Ib) als TRAs (IIc) [5]. (Die Graduierungssysteme sind in den verschiedenen Leitlinien unterschiedlich, siehe dazu die jeweiligen Publikationen.) Die höhere Bewertung der Splenektomie in der ASH-Leitlinie wird damit begründet, dass allein die Splenektomie eine hohe Zahl dauerhafter Remissionen erreicht. Mit TRAs gelingt dies nicht. Unter TRAs werden die Thrombozyten zwar angehoben, aber nur solange diese Medikamente gegeben werden. Wenn die TRAs abgesetzt werden, fallen auch die Thrombozytenzahlen in der Regel wieder ab. Eine dauerhafte Remission ohne ständige Einnahme dieser Medikamente ist die Ausnahme (siehe dazu auch Remissionen unter TRAs). 
Die Möglichkeit der medikamentösen Zweitlinientherapie mit Romiplostim, Eltrombopag oder Rituximab vor Splenektomie sollte mit dem Patienten besprochen werden. Die fehlende arzneimittelrechtliche Zulassung entbindet den Arzt nicht von der Verpflichtung, diese Therapiealternativen gegenüber dem Patienten zu erwähnen. Dabei muss der Patient darauf hingewiesen werden, dass sich die Erfahrung mit diesen Therapien im Vergleich zur Splenektomie auf einen noch relativ kurzen Beobachtungszeitraum beschränken (im Mittel 2-2,5 Jahre), sodass möglicherweise bisher nicht alle Nebenwirkungen bekannt sind. Die Patienten sind auf den fehlenden Zulassungsstatus (off-label) für TRAs (Tab. 8) und Rituximab vor Splenektomie hinzuweisen und über Nebenwirkungsrisiken entsprechend ausführlicher aufzuklären. Der Patient hat keinen Kostenerstattungsanspruch bei Off-label-Therapien.

\section{Drittlinientherapie nach Splenektomie}

Ein Drittel der Patienten erleidet nach Splenektomie einen Rückfall, der eine erneute Therapie notwendig macht [39-41]. Dann sollte mit Sonographie, Computertomographie (CT) und Szintigraphie zunächst eine akzessorische Milz ausgeschlossen werden.

\section{Thrombopoietinrezeptor-Agonisten}

\section{Therapie mit TRAs}

Bei ITP-Patienten sind die Thrombopoietinspiegel im Vergleich zu gesunden Menschen zwar erhöht, aber nicht so hoch wie bei anderen Erkrankungen mit vergleichbarem Thrombozytenmangel. Man spricht deshalb von einem relativen Thrombopoietinmangel [14]. Romiplostim (Nplate ${ }^{\circledR}$ ) ist seit 2009 in der EU zur Therapie der ITP zugelassen, Eltrombopag (Handelsname in Europa Revolade ${ }^{\circledR}$, in den USA und anderen Ländern heißt das Präparat Promacta ${ }^{\mathrm{TM}}$ ) seit 2010.

Die Entscheidung zur Therapie mit TRAs sollte sich primär an der Blutungsneigung und nicht ausschließlich an der Thrombozytenzahl orientieren.

Bei splenektomierten und weiterhin thrombozytopenen Patienten mit WHO Grad-II- bis Grad-IV-Blutungen besteht eine klare Indikation für die Therapie mit TRAs (Empfehlungsgrad A).

Bei splenektomierten, thrombozytopenen Patienten mit geringer oder gänzlich fehlender Blutungsneigung (WHO Grad 0, I) ist der Nutzen einer TRA-Therapie nicht gesichert (Empfehlungsgrad B). Die Erfahrung zeigt jedoch, dass sich viele Patienten angesichts der als bedrohlich empfundenen Thrombozytopenie bei gleichzeitig guter Verträglichkeit von TRAs für diese Therapie entscheiden. Eine Watch-and-WaitStrategie wäre aber bei diesen oligo- oder asymptomatischen Patienten grundsätzlich auch vertretbar. Nutzen und Risiken sollten mit dem Patienten besprochen und eine individuelle Entscheidung sollte gefunden werden.

Romiplostim wird als subkutane Injektion 1-mal pro Woche, Eltrombopag täglich oral verabreicht (Tab. 7). Der Hersteller empfiehlt, Eltrombopag erst $4 \mathrm{~h}$ nach einer kalzium-, magnesium-, aluminium- oder eisenhaltigen Mahlzeit (Antazida, Milchprodukte, Vitamintabletten, Eisensubstitution bei Anämie) einzunehmen. Die Einnahme auf nüchternen Magen oder zur Nacht, vor dem Zubettgehen, ist für viele Patienten am besten praktikabel. Patienten asiatischer Herkunft sprechen stärker auf Eltrombopag an, hier muss eine niedrigere Anfangsdosierung gewählt werden.

Die TRAs Romiplostim und Eltrombopag erreichen bei etwa $80 \%$ der bisher therapierefraktären Patienten einen Anstieg der Thrombozytenzahl über $50 \times 10^{9} / 1$. Ernsthafte Blutungen treten unter TRAs seltener auf $[49,50]$. Die meisten Patienten können ihre bisherige immunsuppressive Medikation, zumeist Steroide, reduzieren oder ganz absetzen.

Die häufigsten Nebenwirkungen von TRAs sind Kopf-, Gelenk-, Muskelschmerzen und Magenbeschwerden. Wichtige weitere Nebenwirkungen von TRAs sind:

- Beim abrupten Absetzen von Romiplostim kann es zu einem überschießenden Abfall der Thrombozytenzahl unter die Ausgangswerte kommen. Deshalb soll nach dem Absetzen von Romiplostim die Thrombozytenzahl noch für mindestens 2 Wochen nachkontrolliert werden. Dies wurde bei Eltrombopag bisher nicht beschrieben.

- Sowohl unter der Behandlung mit Romiplostim als auch mit Eltrombopag werden Thrombosen beobachtet. Retrospektive Studien haben jedoch gezeigt, dass ITP-Patienten auch ohne TRAs ein erhöhtes Thromboserisiko tragen. Insofern muss der Kausalzusammenhang offen bleiben [5153]. Bei Patienten mit Thrombosen in der Vorgeschichte sollte die Indikation zur Gabe von TRAs kritisch gestellt werden. Ein Antiphospholipid-Syndrom als Ursache der ITP muss ausgeschlossen sein.

- Bei Patienten mit chronischer Lebererkrankung (z.B. Zirrhose) wurden Portalvenenthrombosen beschrieben.

- Bei Eltrombopag wurden Leberwerterhöhungen berichtet. Während der Behandlung mit Eltrombopag sollten regelmäßig die Leberwerte kontrolliert werden.

- Bei Eltrombopag wurden Hautveränderungen (Juckreiz, Rötung, makulopapulöses Exanthem) beschrieben [54]. Dann kann alternativ Romiplostim gegeben werden.

- Im Tierversuch wurden unter Eltrombopag vermehrt Katarakte beobachtet. Beim Menschen treten sowohl unter Eltrombopag als auch mit Placebo Katarakte auf. Es könnte durchaus sein, dass dies auf andere Ursachen wie z.B. Steroide zurückzuführen ist [55]. Es wird empfohlen, dass sich Patienten vor Verordnung und auch regelmäßig im weiteren Verlauf augenärztlich untersuchen lassen.

- Bei Patienten mit myelodysplastischen Syndromen, die Romiplostim erhalten haben, wurde eine Blastenvermehrung beschrieben. Deshalb sollte gerade bei älteren Patienten 
vor Gabe von Romiplostim eine Knochenmarkpunktion erwogen werden, um ein myelodysplastisches Syndrom auszuschließen.

- Es liegen Einzelbefunde vor, die bei einigen wenigen Patienten eine leichtgradige Retikulinfaservermehrung im Knochenmark zeigen. Aufgrund der bisher nur sehr begrenzten Daten ist es nicht möglich abzuschätzen, wie häufig die Dauertherapie mit Thrombozyten-Wachstumsfaktoren eine klinisch relevante Knochenmarksfibrose auslösen kann. Eine regelmäßige Kontrolle des Blutausstriches auf Veränderungen, die auf eine beginnende Knochenmarksfibrose hinweisen könnten (Normoblasten, «Tear-Drop»Zellen), wird empfohlen [56, 57]. Bei auffälligen Befunden wird eine Knochenmarkpunktion empfohlen.

- Bei 3 Patienten mit Romiplostim wurden bisher neutralisierende Anti-Romiplostim-Antikörper beobachtet, die nach dem Absetzen von Romiplostim wieder verschwanden $[58,59]$. Für Eltrombopag ist dergleichen bisher nicht beschrieben $[60,61]$.

- Die bisherigen Langzeitbeobachtungsstudien finden keine neuen, unerwarteten Sicherheitsrisiken [61-64].

\section{Remissionen unter TRAs}

Es wurden Einzelfälle beschrieben, bei denen nach dem Absetzen von TRAs die Thrombozytenzahl nicht wieder abgefallen ist $[49,65,66]$. Es muss offen bleiben, ob durch die erhöhte Thrombozytenzahl unter TRAs eine Desensibilisierung und Herunterregulierung des gegen sie gerichteten Immunprozesses erreicht wurde oder ob es sich nur um zufällige Spontanremissionen handelt, die auch ohne TRA-Therapie aufgetreten wären.

\section{Rituximab}

Rituximab ist in keinem Land der Welt zur Behandlung der ITP zugelassen, dennoch wird es allgemein als eine wirksame Therapie bei chronischer ITP betrachtet und in den Leitlinien empfohlen [1-5] (Empfehlungsgrad B). Rituximab erreicht bei etwa $30 \%$ der chronischen ITP-Patienten langfristige - definiert als über 12 Monate anhaltende - Remissionen [48, 67]. Die übliche Dosis liegt bei $375 \mathrm{mg} / \mathrm{m}^{2} 1 \times$ pro Woche für 4 aufeinanderfolgende Wochen. Es ist bisher nicht geklärt, ob niedrigere Dosen gleich wirksam sind [68]. Die Behandlung mit Rituximab ist in der Regel gut verträglich. Es wurden aber auch ernste Nebenwirkungen berichtet, insbesondere Infektionen und Einzelfälle von progressiver multifokaler Leukoenzephalopathie [69]. Es muss offen bleiben, inwieweit diese auf Rituximab oder die immunsuppressive Vor- und Begleittherapie zurückzuführen waren. Vor der Gabe von Rituximab sollte eine Virushepatitis ausgeschlossen werden (Empfehlungsgrad B).

\section{Weitere Drittlinientherapien}

Die weiteren Therapieoptionen kommen in der Regel nur dann infrage, wenn Splenektomie, TRAs und auch Rituximab kein ausreichendes Ansprechen der Blutungsneigung erreichen:

- Azathioprin

- Cyclophosphamid

- Ciclosporin A

- Danazol

- Dapson

- Mycophenolat-Mofetil

- Vincristin/Vinblastin

Bei der Auswahl der Therapie besteht keine Präferenz, die Entscheidung muss individuell getroffen werden (Tab. 7). Man sollte berücksichtigen, dass Azathioprin und Vinca-Alkaloide nur eine sogenannte «Altzulassung» zur Behandlung der ITP haben. Der Anteil dauerhafter Remissionen ist nicht sehr hoch, dafür sind die Nebenwirkungen zum Teil erheblich. Sie sollten deshalb nur gegeben werden, wenn TRAs und Rituximab nicht wirksam sind (Empfehlungsgrad B). Außerdem müssen die Nebenwirkungen der Therapie immer gegen den Nutzen abgewogen werden. In späteren Therapielinien ist es kein Fehler, auch bei niedrigsten Thrombozytenwerten eine Watch-and-Wait-Strategie zu verfolgen, solange der Patient nicht oder nur minimal blutet.

Weitere experimentelle Therapieverfahren oder Fallberichte

- Alemtuzumab/Campath $1 \mathrm{H}$

- Kombination mehrerer Zytostatika oder Immunsuppressiva

- Stammzelltransplantation

\section{Unwirksame oder nicht indizierte Therapien}

Folgende Therapien sind unwirksam und sollten nicht mehr eingesetzt werden [4]:

- Colchicin

- Interferon $\alpha$ (Ausnahme: zur Therapie einer Virushepatitis mit sekundärer ITP)

- Protein-A-Immunadsorption

- alleinige Plasmapherese

- Vitamin C

\section{Antifibrinolytika}

Durch die Gabe des Fibrinolyseinhibitors Tranexamsäure (Cyklokapron ${ }^{\circledR}$ : Filmtabletten à $500 \mathrm{mg}, 20-25 \mathrm{mg} / \mathrm{kg}$ alle $8 \mathrm{~h}$ oral; Injketionslösung $0,5-1 \mathrm{~g}$ (Ampullen à 5 oder $10 \mathrm{ml}$ ) alle 8-12 h langsam intravenös (die Injektionslösung ist $~ 10 \%$ ig, 
Tab. 10. Anzustrebende Thrombozytenwerte vor operativen Eingriffen (Empfehlungsgrad B)
Zahnärztliche Zahnreinigung, Zahnsteinentfernung

Zahnextraktion (einfach)

Zahnextraktion (komplex, z.B. molar)

Leitungsanästhesie bei Zahneingriff

Lumbalpunktion

Spinalanästhesie

Epiduralanästhesie

Gastrointestinale Endoskopie ohne Biopsie

Gastrointestinale Endoskopie mit Biopsie

Bronchoskopie/Bronchiallavage

Bronchoskopie mit transbronchialer Biopsie

Andere Organpunktionen/Biopsien

Kleine Operation ${ }^{\mathrm{a}}$

Größere Operation ${ }^{\mathrm{a}}$

Neurochirurgischer Eingriff

Eingriffe am hinteren Augenabschnitt

Knochenmarkbiopsie
$>20 \times 10^{9}-30 \times 10^{9} / 1$

$>30 \times 10^{9} / 1$

$>50 \times 10^{9} / 1$

$>30 \times 10^{9} / 1$

$>50 \times 10^{9} / 1$

$>50 \times 10^{9} / 1$

$>80 \times 10^{9} / 1$

auch bei sehr niedrigen Werten möglich

$>20 \times 10^{9} / 1$

$>20 \times 10^{9} / 1$

$>50 \times 10^{9} / 1$

$>50 \times 10^{9} / 1$

$>50 \times 10^{9} / 1$

$>80 \times 10^{9} / 1$

$>70 \times 10^{9}-100 \times 10^{9} / 1$

$>70 \times 10^{9}-100 \times 10^{9} / 1$

auch bei sehr niedrigen Werten möglich

Diese Zahlen wurden für Patienten mit Thrombozytenbildungsstörungen erhoben. Für ITP-Patienten gibt es keine entsprechenden Daten. Hier muss auch die individuelle Blutungsanamnese berücksichtigt werden, d.h. ob ein Patient in seiner Vorgeschichte bereits bei den angegebenen Thrombozytenzahlen geblutet hat.

${ }^{\text {a }}$ Größere Operationen sind z.B. abdominelle oder thoraxchirurgische Eingriffe und Operationen in Regionen, die im Falle einer postoperativen Blutung nicht komprimiert werden können.

Verdünnung mit gleichem Volumen ergibt eine 5\%ige Lösung); $5 \%$ ige Tranexam-Mundspüllösung $4 \times$ täglich) kann bei leichten oralen Schleimhautblutungen, bei Menorrhagien und im Rahmen von Zahneingriffen häufig eine ausreichende Blutstillung erreicht werden (Empfehlungsgrad B). Bei Blutungen in das Auge, das Zentralnervensystem (ZNS) oder bei Organblutungen sind Antifibrinolytika nicht ausreichend wirksam.

\section{Zweit- und Drittlinientherapien in der Kinder- und Jugendmedizin}

In der Pädiatrie existieren bisher keine systematischen Daten zur Therapie protrahierter oder chronischer Verläufe. Die Patienten sollten in Zentren mit pädiatrisch hämatologischer Expertise betreut werden (Empfehlungsgrad B). Studien zu TRAs sind aktiviert. Ihr Einsatz ist in der Pädiatrie derzeit noch mit kritischer Distanz zu bewerten, da Informationen über Langzeitnebenwirkungen beim Einsatz an Kindern noch nicht vorliegen.

Eine Splenektomie kann als Ultima Ratio diskutiert werden. In einzelnen Zentren liegen Erfahrungen mit verschiedenen immunsupressiven Konzepten vor. Ein Standard kann nicht formuliert werden.

In der Pädiatrie hat sich die antifibrinolytische Therapie mit Tranexamsäure insbesondere bei Schleimhautblutungen bewährt.

\section{Vorgehen bei Operationen und Zahneingriffen}

Wenn bei Patienten mit ITP eine Operation oder ein invasiver diagnostischer Eingriff geplant wird, dann stellt sich die Frage nach den präoperativ anzustrebenden Thrombozytenwerten (Tab. 10) [70, 71]. Bei Notfalloperationen wird man in der Regel Steroide mit intravenös applizierten Immunglobulinen kombinieren. In allen anderen Fällen, insbesondere bei elektiven Operationen, erreichen Kortikosteroide meist innerhalb von 1-2 Wochen eine ausreichende Thrombozytenzahl.

\section{Impfungen}

Kurzfristige Thrombozytopenien in Assoziation mit Impfungen wurden beschrieben (nach Masern-Röteln-MumpsImpfung: 1:40 000). Fälle von chronischer ITP nach Impfungen sind jedoch so selten, dass es sich auch um zufällige Assoziationen handeln könnte. Aktuelle Studien finden keine Häufung chronischer ITP nach Impfungen [72]. Bei den seltenen Patienten, bei denen ein Zusammenhang zwischen ITP und einer vorhergehenden Impfung vermutet wird, sollten weitere Impfungen mit diesem oder anderen Impfstoffen, die ähnliche Bestandteile enthalten, vermieden werden.

Es gibt keine Untersuchungen, ob bei Patienten, die eine ITP in der Anamnese haben und die jetzt in Remission sind, oder bei Patienten, die aktuell unter einer chronischen ITP leiden, eine Impfung einen Rückfall oder eine Verschlimmerung der Thrombozytopenie hervorrufen kann. Wenn die Imp- 
Tab. 11. Differenzialdiagnose der Thrombozytopenien in der Schwangerschaft
Schwangerschaftsspezifische Thrombozytopenien

\begin{tabular}{ll} 
& Gestationsthrombozytopenie \\
& HELLP-Syndrom \\
& Verbrauchskoagulopathie \\
& akute Schwangerschaftsfettleber \\
& Eklampsie \\
\hline Nicht schwangerschaftsspezifische Thrombozytopenien & ITP \\
& SLE \\
& TTP/HUS \\
& Virusinfektionen \\
& Knochenmarkserkrankungen, hämatologische Neoplasien \\
& Medikamente \\
& Hypersplenismus bei Lebererkrankungen, unter anderem
\end{tabular}

SLE = Systemischer Lupus erythematodes, TTP = thrombotisch-thrombozytopenische Purpura, HUS = hämolytischurämisches Syndrom. fung unterlassen wird und der Patient dann an der Infektion erkrankt, hat er möglicherweise ein höheres Risiko, dass diese Infektion die Thrombozytopenie verschlimmert [73]. Jeder Patient sollte zur Grippe- und Pneumovax-Impfung ermutigt werden.

Lebendimpfungen sind bei immunsupprimierten Patienten in der Regel kontraindiziert (Anmerkung: der nasale Influenza-Impfstoff Fluenz ${ }^{\circledR}$ ist ein Lebendimpfstoff mit attenuierten Viren).

ITP-Patienten, bei denen aufgrund häufiger oder schwerer Blutungen bzw. bei Therapieresistenz eine baldige Splenektomie zu den näherliegenden Therapieoptionen zählt, sollten frühzeitig gegen Pneumokokken, Meningokokken und Haemophilus influenzae B geimpft werden.

Bei allen ITP-Patienten empfiehlt sich eine Hepatitis-BImpfung, weil eine Bluttransfusion oder die Gabe von anderen Blutprodukten und das damit verbundene Infektionsrisiko für die Zukunft nie ganz ausgeschlossen werden können.

\section{ITP und Schwangerschaft}

Die Häufigkeit einer ITP in der Schwangerschaft beträgt etwa 1:1000-1:10 000 [4, 5, 74]. Das ungeborene Kind einer Schwangeren mit ITP hat ein geringes Risiko, durch den plazentaren Übertritt von Thrombozyten-Antikörpern selber eine Thrombozytopenie zu entwickeln. Das Risiko einer Thrombozytopenie beim Neugeborenen liegt bei etwa 5-10\%. Intrazerebrale Blutungen treten bei $<1,5 \%$ auf und die neonatale Mortalität beträgt $<1 \%$. Die Blutungen manifestieren sich nicht wie bei der neonatalen Allo-ITP bereits intrauterin, sondern in der Regel perinatal.

Die mütterlichen Thrombozytenwerte korrelieren nicht mit denen des Kindes. Dies ist insbesondere bei splenektomierten Frauen zu beachten. Dann können die Thrombozytenwerte der Mutter gering erniedrigt oder sogar normal sein, während der Antikörper, der durch die Splenektomie nicht verschwunden ist, beim Kind eine viel stärkere Thrombozytopenie verursacht. Wenn bereits bei einer vorhergehenden Geburt eine Thrombozytopenie des Neugeborenen aufgetreten war, dann ist die Wahrscheinlichkeit hoch, dass sich dies bei der nächsten Geburt wiederholt. Andere Marker zur Vorhersage des kindlichen Risikos gibt es nicht. Die Differenzialdiagnosen schwangerschaftsassoziierter Thrombozytopenien sind in Tabelle 11 aufgeführt.

Die Therapie einer ITP in der Schwangerschaft bereitet besondere Probleme, da die notwendigen Medikamente Nebenwirkungen auf die Schwangerschaft und den Fötus haben können.

\section{Therapieindikation in der Schwangerschaft}

Eine Therapieindikation besteht

- bei Blutungen,

- zur Vorbereitung invasiver Eingriffe (z.B. Sectio, Periduralanästhesie),

- bei Thrombozyten unter $20 \times 10^{9}-30 \times 10^{9} / 1$ im ersten und zweiten Trimenon,

- bei Werten unter $50 \times 10^{9} / 1$ im letzten Trimenon. Für eine Sectio wird eine Thrombozytenzahl über $50 \times 10^{9} / 1$ als ausreichend angesehen, für eine Periduralanästhesie Werte von $80 \times 10^{9}-100 \times 10^{9} / 1$.

\section{Therapieoptionen}

- Steroide (Predniso(lo)n). Man wird - wenn kein Notfall die Verwendung höherer Dosen erforderlich macht - mit einer Dosis von 20-30 mg/Tag beginnen und versuchen, diese rasch so weit zu reduzieren, dass eine Thrombozytenzahl von $20-30 \times 10^{9} / 1$ gehalten werden kann (meist reichen 10-20 mg/Tag) (Empfehlungsgrad B).

- Wenn höhere Steroiddosen notwendig sind oder wenn Nebenwirkungen auftreten (Hypertonie, diabetische Stoffwechsellage, Osteoporose, starker Gewichtsanstieg, Psychose usw.) sollte man alternativ intravenöse Immunglobuline anwenden. Immunglobuline können wiederholt und 
Abb. 2. Therapiealgorithmus zur Antikoagulation bei ITP.
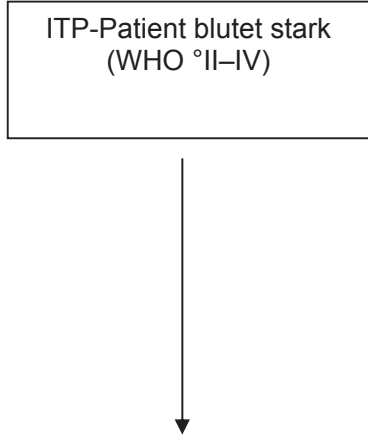

Antikoagulation kontraindiziert

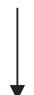

ITP-Patient blutet nicht oder nur mäßig $\left(\mathrm{WHO}{ }^{\circ} / \mathrm{l}\right)$
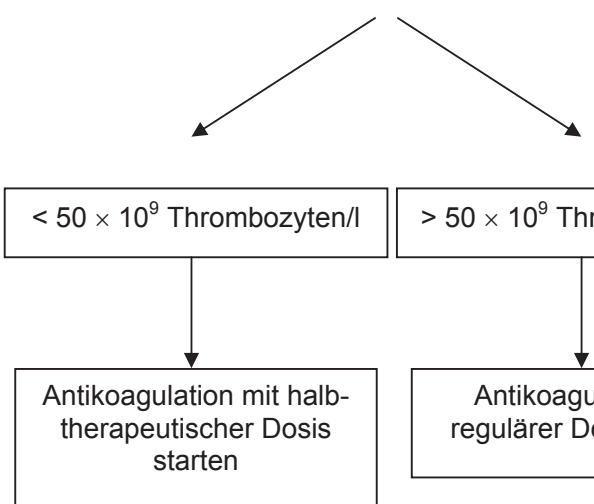

$>50 \times 10^{9}$ Thrombozyten/l
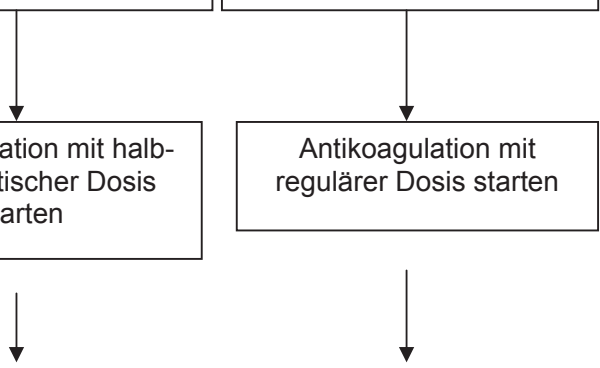

Im weiteren Verlauf Antikoagulation an Blutungsneigung anpassen: Antikoagulation stoppen oder reduzieren, wenn sich Blutungen verschlimmern. Antikoagulation ggf. von halbtherapeutischer auf volle therapeutische Dosis anheben, wenn gar keine Blutungen eintreten.

Bei venösen Thromboembolien Cavafilter erwägen.

besonders zum Ende der Schwangerschaft zur Vorbereitung der Entbindung gegeben werden (Empfehlungsgrad $B)$.

- Bei schwerer, nicht anders kontrollierbarer Thrombozytopenie und Blutungen ist die Splenektomie indiziert. Wenn möglich sollte die Splenektomie laparoskopisch im zweiten Trimester durchgeführt werden (Empfehlungsgrad B).

- Azathioprin kann in der Schwangerschaft eingesetzt werden (Empfehlungsgrad 0).

- Ciclosporin wurde bei Patientinnen mit anderen Immunerkrankungen (Morbus Crohn, Colitis) in der Schwangerschaft eingesetzt, es gibt keine Daten zur ITP.

- Vincristin, Cyclophosphamid und Danazol sind in der Schwangerschaft kontraindiziert [75].

- Es gibt kaum Erfahrungen zu TRAs in der Schwangerschaft. Den Autoren sind bisher nur zwei Fallberichte bekannt $[76,77]$. Eine Schädigung des Fötus ist nicht auszuschließen. Auch während der Stillzeit sollten TRAs nicht genommen werden (gegebenenfalls abstillen). Bei ITP-Patientinnen mit starkem Kinderwunsch, die noch nicht splenektomiert wurden und die bisher nur auf TRAs ansprechen, muss die Möglichkeit der Milzentfernung vor der Schwangerschaft diskutiert werden.

\section{Peri- und postpartales Management}

Früher wurde ITP-Patientinnen die Sectio empfohlen, unter der Annahme, dass dadurch das Geburtstrauma und das Blutungsrisiko für das Kind unter der Geburt geringer seien als bei einer vaginalen Entbindung. Es gibt jedoch keinerlei Daten, die dies unterstützen. Die Entscheidung sollte nicht durch die ITP, sondern allein durch die geburtshilfliche Situation der Mutter bestimmt werden. Dies spricht jedoch nicht gegen eine aktive Einleitung der Geburt aus organisatorischen Gründen, um das peripartale Monitoring und die hämatologisch-hämostaseologische Mitbetreuung optimal zu gewährleisten (Entbindung, wenn die Thrombozyten nach vorbereitender intravenöser Immunglobulingabe am höchsten sind, keine Entbindung freitags oder am Wochenende).

Eine Blutentnahme aus der Nabelschnur (Cordozentese) vor der Entbindung, um die Thrombozytenzahl des Fötus zu bestimmen, ist bei Patientinnen mit chronischer ITP nicht sinnvoll. Die betroffenen Kinder erleiden ihre Blutungen - im Gegensatz zur neonatalen Allo-ITP - nicht während der Schwangerschaft, sondern fast ausschließlich nach der Geburt. Unmittelbar nach der Entbindung sollte die kindliche Thrombozytenzahl bestimmt und ein transkranieller Ultraschall durchgeführt werden. Hat das Neugeborene $<20 \times 10^{9}$ 
Thrombozyten/l oder bei Blutungszeichen, dann kann mit intravenösen Immunglobulinen und Steroiden behandelt werden. Der Thrombozytennadir kann auch erst nach einigen Tagen auftreten (bis zu 1 Woche nach der Geburt), entsprechend lang sollte die Thrombozytenzahl kontrolliert werden.

Postpartal hat die Mutter durch die präpartale ITP-Therapie meist Thrombozyten $>50 \times 10^{9} / 1$ oder sogar Werte im Normbereich. Da die ITP eine «thrombophile» Erkrankung ist [51], sollte bei diesen Werten und Immobilität der Wöchnerin eine Thromboseprophylaxe erwogen werden (Kompressionsstrümpfe, niedermolekulares Heparin (NMH)).

\section{ITP und Antikoagulation}

Die Hälfte aller ITP-Patienten ist älter als 50 Jahre. Nebenerkrankungen, die eine Antikoagulation erfordern (Thromboembolien, Vorhofflimmern, Herzinfarkt, perkutane transluminale koronare Angioplastie (PTCA) mit Stent, zerebrovaskuläre und peripher-arterielle Ereignisse usw.) treten dann häufiger auf. Antikoagulanzien sind bei Thrombozytopenie in der Regel kontraindiziert. Für diese klinisch immer häufigeren Situationen gibt es nur Fallberichte, die keine evidenzbasierte Empfehlung erlauben [78-81].

Die Autoren empfehlen bei dringender Indikation zur therapeutischen Antikoagulation folgendes Vorgehen (Abb. 2). Die Kernpunkte der Empfehlungen können folgendermaßen zusammengefasst werden (Empfehlungsgrad 0):

- Eine Antikoagulation ist bei thrombozytopenen Patienten mit ITP kontraindiziert, wenn diese bedrohlich bluten (WHO Grad II-IV). Bei venösen Thromboembolien kann alternativ ein Cavafilter erwogen werden.

- Eine Antikoagulation ist nicht kontraindiziert bei Patienten mit ITP, die nicht oder nur mild bluten (WHO Grad $0-\mathrm{I})$ :

- Thrombozyten $\geq 50 \times 10^{9} /$ l: volldosierte Antikoagulation.

- Thrombozyten < $50 \times 10^{9} / 1$ : Verminderung der Dosis, z.B. halbtherapeutische Antikoagulation.

- Parallel zur notwendigen Antikoagulation bei niedrigen Thrombozytenwerten Anhebung der Thrombozytenzahl mit Steroiden und intravenösen Immunglobulinen über $50 \times 10^{9} / 1$.

- Gleichzeitig Start von TRAs, die für ihren Wirkungseintritt meist $>1$ Woche brauchen.

- Sobald die Thrombozyten auf $>50 \times 10^{9} / 1$ angestiegen sind, Ausschleichen der Steroide und Anpassung der TRADosis.

- Weitere Anpassung der Antikoagulationsdosis entsprechend des Anstiegs der Thrombozytenzahl und der klinischen Blutungsneigung.

Man kann statt mit einem NMH auch mit unfraktioniertem Heparin (UFH) beginnen, z.B. in Form eines Heparin-Perfusors. UFH hat eine kürzere Halbwertszeit, es kann mit der aktivierten partiellen Thromboplastinzeit (aPTT) gut überwacht, bei Überdosierung schnell gestoppt und gegebenenfalls sogar antagonisiert werden. Wenn unter UFH keine Blutungen auftreten, kann man im weiteren Verlauf auf ein NMH umstellen.

\section{Evidenzgraduierung und Empfehlungsgrade}

Die Evidenzgraduierung folgt der Empfehlung des Oxford Centre of Evidence-Based Medicine (www.cebm.net/index. aspx? $=1025$, Levels of Evidence, Version März 2009).

Die Festlegung der Empfehlungsgrade A, B oder 0 folgt den Empfehlungen des Nationalen Programms für die Versorgungsleitlinien ( $w w w . v e r s o r g u n g s l e i t l i n i e n . d e / m e t h o d i k / p d f$ ) $\left.n v l \_m e t h o d e \_4 . a u f l . p d f\right)$ :

- A: Starke Empfehlung (soll)

- B:Empfehlung (sollte)

- 0: Empfehlung offen (kann)

\section{Links zu Selbsthilfegruppen (SHG)}

ITP-SHG Gießen: www.itp-information.de, Kontakt: Fr. Arnold (über Homepage)

ITP-SHG Hamburg: www.morbus-werlhof.de, Kontakt: Fr. Hass (über Homepage)

ITP-SHG Sömmerda: Kontakt: Fr. Riese (s-riese@t-online.de)

USA: Platelet Disorder Support Organisation: www.pdsa.org Großbritannien: ITP Support Association: www.itpsupport. org.uk

\section{Abschließende Stellungnahme}

Die Autoren beschreiben in dieser Leitlinie den aktuellen Kenntnisstand in der Behandlung der ITP. Sämtliche Aussagen sind ausschließlich als Hilfen zur ärztlichen Entscheidungsfindung intendiert und nicht geeignet, einen medizinischen Standard im sozial- oder strafrechtlichen Sinne zu definieren.

Die Autoren übernehmen keine Gewähr und Haftung für die Richtigkeit, Vollständigkeit, Aktualität, Qualität und jederzeitige Verfügbarkeit der Textinhalte und Quellenangaben. Haftungsansprüche gegen die Autoren, die sich auf Schäden materieller oder ideeller Art beziehen, die durch die Nutzung oder Nichtnutzung der dargebotenen Informationen bzw. durch die Nutzung fehlerhafter und unvollständiger Informationen verursacht wurden, sind ausgeschlossen. Soweit Gesetze, Normen, Verordnungen oder Ähnliches zitiert werden, übernehmen die Autoren keine Gewähr für die Richtigkeit bzw. Aktualität. In Zweifelsfällen sind die Originalquellen heranzuziehen. Für Angaben über Verfahrensweisen oder Anwendungen, Applikationsformen und Dosierungen wird von den Autoren keine Gewähr übernommen. Jeder Benutzer ist angehalten, z.B. durch Lesen der Gebrauchsanweisung von 
medizinisch-technischen Geräten oder durch das Studium des Beipackzettels von verwendeten Präparaten und gegebenenfalls zusätzlicher Konsultation eines Spezialisten oder weiterer Literatur festzustellen, ob die dort angegebene Empfehlung zur Anwendung bzw. zur Dosierung oder die Beachtung von Kontraindikationen gegenüber den Angaben in dieser Leitlinie abweicht. Jede Anwendung, Applikation oder Dosierung erfolgt auf eigene Gefahr des Benutzers. Die Angaben in dieser Leitlinie sind kein Ersatz für eine individuelle professionelle Beratung oder Behandlung durch ausgebildete und anerkannte Fachleute.

\section{Disclosure Statement}

A. Matzdorff: Beratungstätigkeit: AMGEN, Baxter, GlaxoSmithKline; Besitz von Geschäftsanteilen, Aktien oder Fonds: Roche-Aktien in Familienbesitz; Referentenhonorare: AMGEN, Behring, GlaxoSmithKline, Roche; Finanzierung wissenschaftlicher Untersuchungen: Roche; Gutachtertätigkeit: AOK; Mitarbeiterweiterbildungen, Reisekosten, usw.: GSK, Roche, AMGEN. W. Eberl: Beratungstätigkeit: CSL-Behring; Referentenhonorare: Bayer AG, Baxter, Octapharma, Novo Nordisk, CSL-Berking. A. Giagounidis: Beratungstätigkeit: AMGEN, GlaxoSmithKline; Referentenhonorare: AMGEN, GlaxoSmithKline; Mitarbeiterweiterbildungen, Reisekosten, usw.: AMGEN. P. Imbach: Keine Interessenkonflikte. I. Pabinger: Beratungstätigkeit: AMGEN, Baxter, GlaxoSmithKline, CSL Behring; Referentenhonorare: AMGEN, CSL Behring, GlaxoSmithKline, Roche; Reisekostenunterstützung: AMGEN, CSL Behring. B. Wörmann: Keine Interessenkonflikte.

\section{Literatur}

1 Matzdorff A, Giagounidis A, Greinacher A, Hiller E, Kiefel V, Müller-Beißenhirtz H, Ostermann H, Rummel M, Sachs UJ, Salama A: Diagnostik und Therapie der Immunthrombozytopenie. Empfehlungen einer gemeinsamen Expertengruppe der DGHO, DGTI und GTH. Onkologie 2010;33 (suppl 3):2-20.

2 Pabinger I, Gastl G, Steurer M, Sormann S, Fillitz M, Friedl J, Geissler D, Geissler K, Greil R, Knöbl P, Kozek-Langenecker S, Krippl P, Kyrle P, Lang A, Linkesch W, Ludwig H, Müller M, Panzer S, Pittermann E, Thaler J, Weltermann A: Primäre Immunthrombozytopenie des Erwachsenen - Diagnostik und Therapie, Konsensus-Statement der Österreichischen Gesellschaft für Hämatologie und Onkologie (ÖGHO). Wien Klin Wochenschr 2012;124:111-123.

3 Keller P, Angelillo-Scherrer A, Bargetzi M, Goede J, Hess U, Imbach P, Kühne T, Mach-Pascual S, Mey U, Rüfer A, Schmidt A: Diagnose und Behandlung der primären Immun-Thrombozytopenie. Ein Schweizer Konsensus, erarbeitet von Hämatologen der grösseren hämatologischen Zentren der Schweiz. Schweiz Med Forum 2010;10:875-883.

- 4 Provan D, Stasi R, Newland AC, Blanchette VS, Bolton-Maggs P, Bussel JB, Chong BH, Cines DB, Gernsheimer TB, Godeau B, Grainger J, Greer I, Hunt BJ, Imbach PA, Lyons G, McMillan R, Rodeghiero F, Sanz MA, Tarantino M, Watson S, Young J, Kuter DJ: International consensus report on the investigation and management of primary immune thrombocytopenia. Blood 2010;115:168-186.

$\checkmark 5$ Neunert C, Lim W, Crowther M, Cohen A, Solberg L Jr, Crowther MA; American Society of Hematology: The American Society of Hematology 2011 evidence-based practice guideline for immune thrombocytopenia. Blood 2011;117:4190-4207.

6 Dickerhoff R, Eberl W: Leitlinie «Immunthrombozytopenie (ITP) im Kindes- und Jugendalter», Stand 8/2011, AWMF-Register Nr. 086/001, Klasse S2k.www.awmf.org/leitlinien/detail/ll/086-001.html.

7 Rodeghiero F, Stasi R, Gernsheimer T, Michel M, Provan D, Arnold DM, Bussel JB, Cines DB, Chong BH, Cooper N, Godeau B, Lechner K, Mazzucconi MG, McMillan R, Sanz MA, Imbach P, Blanchette V, Kühne T, Ruggeri M, George JN: Standardization of terminology, definitions and outcome criteria in immune thrombocytopenic purpura (ITP) of adults and children: report from an international working group. Blood 2009;113: 2386-2393.
8 Cines DB, Bussel JB, Liebman HA, Prak ETL: The ITP syndrome: pathogenic and clinical diversity. Blood 2009:113:6511-6521.

9 Chang M, Nakagawa PA, Williams SA, Schwartz MR, Imfeld KL, Buzby JS, Nugent DJ: Immune thrombocytopenic purpura (ITP) plasma and purified ITP monoclonal autoantibodies inhibit megakaryocytopoiesis in vitro. Blood 2003;102:887-895.

10 McMillan R, Wang L, Tomer A, Nichol J, Pistillo J: Suppression of in vitro megakaryocyte production by antiplatelet autoantibodies from adult patients with chronic ITP. Blood 2004;103:1364-1369.

11 Li X, Zhong H, Bao W, Evangelista J, Haider MA, Bussel J, Yazdanbakhsh K: Defective regulatory Bcell compartment in patients with immune thrombocytopenia. Blood 2012;120:3318-3325.

12 Olsson B, Andersson PO, Jernås M, Jacobsson S, Carlsson B, Carlsson LM, Wadenvik H: T-cell-mediated cytotoxicity toward platelets in chronic idiopathic thrombocytopenic purpura. Nat Med 2003; 9:1123-1124.

13 Aslam R, Hu Y, Gebremeskel S, Segel GB, Speck ER, Guo L, Kim M, Ni H, Freedman J, Semple JW: Thymic retention of CD4+CD25+FoxP3+ T regulatory cells is associated with their peripheral deficiency and thrombocytopenia in a murine model of immune thrombocytopenia. Blood 2012;120:21272132.

14 Emmons RV, Reid DM, Cohen RL, Meng G, Young NS, Dunbar CE, Shulman NR: Human thrombopoietin levels are high when thrombocytopenia is due to megakaryocyte deficiency and low when due to increased platelet destruction. Blood 1996;87:4068-4071.

15 Neunert CE, Buchanan GR, Imbach P, BoltonMaggs PH, Bennett CM, Neufeld E, Vesely SK, Adix L, Blanchette VS, Kühne T; Intercontinental Cooperative ITP Study Group Registry II Participants: Bleeding manifestations and management of children with persistent and chronic immune thrombocytopenia: data from the Intercontinental Cooperative ITP Study Group (ICIS). Blood 2013;121:4457-4462.

16 Heddle NM, Cook RJ, Tinmouth A, Kouroukis CT, Hervig T, Klapper E, Brandwein JM, Szczepiorkowski ZM, AuBuchon JP, Barty RL, Lee KA; StoP Study Investigators of the BEST Collaborative: A randomized controlled trial comparing standard and low-dose strategies for transfusion of platelets (StoP) to patients with thrombocytopenia. Blood 2009;113:1564-1573.
17 National Cancer Institute: NCI Common Terminology Criteria for Adverse Events: CTCAE, version MedDRA v9,0. www.cancer.gov.

18 Rodeghiero F, Michel M, Gernsheimer T, Ruggeri M, Blanchette V, Bussel JB, Cines DB, Cooper N, Godeau B, Greinacher A, Imbach P, Khellaf M, Klaassen RJ, Kühne T, Liebman H, Mazzucconi MG, Newland A, Pabinger I, Tosetto A, Stasi R: Standardization of bleeding assessment in immune thrombocytopenia: report from the International Working Group. Blood 2013;121:2596-2606.

19 Sailer T, Lechner K, Panzer S, Kyrle PA, Pabinger I: The course of severe autoimmune thrombocytopenia in patients not undergoing splenectomy. Haematologica 2006;91:1041-1045.

20 Andrès E, Mecilli M, Fothergill H, Zimmer J, Voge T, Maloisel F: Gender-related analysis of the clinical presentation, treatment response and outcome in patients with immune thrombocytopenia. Presse Med 2012;41:e426-e431.

21 Ghanima W, Godeau B, Cines DB, Bussel JB: How I treat immune thrombocytopenia: the choice between splenectomy or a medical therapy as a second-line treatment. Blood 2012;120:960-969.

22 Djulbegovic B, Cohen Y: The natural history of refractory idiopathic thrombocytopenic purpura. Blood 2001;98:2282-2283.

23 Cohen YC, Djulbegovic B, Shamai-Lubovitz O, Mozes B: The bleeding risk and natural history of idiopathic thrombocytopenic purpura in patients with persistent low platelet counts. Arch Intern Med 2000;160:1630-1638.

24 Portielje JE, Westendorp RG, Kluin-Nelemans HC, Brand A: Morbidity and mortality in adults with idiopathic thrombocytopenic purpura. Blood 2001; 97:2549-2554.

25 Nørgaard M, Jensen A $\varnothing$, Engebjerg MC, Farkas DK, Thomsen RW, Cha S, Zhao S, Sørensen HT: Long-term clinical outcomes of patients with primary chronic immune thrombocytopenia: a Danish population-based cohort study. Blood 2011;117: 3514-3520.

26 Frederiksen H, Maegbaek ML, Sorensen HT: Longterm mortality of patients with primary immune thrombocytopenia. Blood 2012;120:abstr 619.

27 Aster RH, Bougie DW: Current concepts: Druginduced immune thrombocytopenia. N Engl J Med 2007;357:580-587. 
28 Arnold DM, Kukaswadia S, Nazi I, Esmail A, Dewar L, Smith JW, Warkentin TE, Kelton JG: A systematic evaluation of laboratory testing for drug-induced immune thrombocytopenia. J Thromb Haemost 2013;11:169-176.

29 Reese JA, Li X, Hauben M, Aster RH: Identifying drugs that cause acute thrombocytopenia: an analysis using 3 distinct methods. Blood 2010;116:2127-2133.

-30 Aster RH: Can drugs cause autoimmune thrombocytopenic purpura? Semin Hematol 2000;37:229-238.

-31 Schoonen WM, Kucera G, Coalson J, Li L, Rutstein M, Mowat F, Fryzek J, Kaye JA: Epidemiology of immune thrombocytopenic purpura in the General Practice Research Database. Br J Haematol 2009; 145:235-244

- 32 Arnold DM, Bernotas A, Nazi I, Stasi R, Kuwana M, Liu Y, Kelton JG, Crowther MA: Platelet coun response to $H$. pylori treatment in patients with immune thrombocytopenic purpura with and without H. pylori infection: a systematic review. Haematologica 2009;94:850-856.

-33 Arnold DM, Santoso S, Greinacher A; on behalf of the Platelet Immunology Scientific Subcommittee of the ISTH: Recommendations for the implementation of platelet autoantibody testing in clinical trials of immune thrombocytopenia. J Thromb Haemost 2012;10:695-697.

-34 Kühne T, Berchtold W, Michaels LA, Wu R, Donato H, Espina B, Tamary H, Rodeghiero F, Chitlur M, Rischewski J, Imbach P; Intercontinental Cooperative ITP Study Group: Newly diagnosed immune thrombocytopenia in children and adults: a comparative prospective observational registry of the Intercontinental Cooperative Immune Thrombocytopenia Study Group. Haematologica 2011;96:1831-1837.

35 Bae SH, Ryoo HM, Lee WS, Joo YD, Lee KH, Lee JH, Lee JH, Kim H, Park JH, Kim MK, Hyun MS, Kim HJ, Zang DY: High dose dexamethasone vs. conventional dose prednisolone for adults with immune thrombocytopenia: a prospective multicenter phase III trial. American Society of Hematology Congress. Blood 2010;116:abstr 3687.

-36 George JN, Woolf SH, Raskob GE, Wasser JS, Aledort LM, Ballem PJ, Blanchette VS, Bussel JB, Cines DB, Kelton JG, Lichtin AE, McMillan R, Okerbloom JA, Regan DH, Warrier I: Idiopathic thrombocytopenic purpura: a practice guideline developed by explicit methods for the American Society of Hematology. Blood 1996;88:3-40.

-37 Salama A, Kiesewetter H, Kalus U, Movassaghi K, Meyer O: Massive platelet transfusion is a rapidly effective emergency treatment in patients with refractory autoimmune thrombocytopenia. Thromb Haemost 2008;100:762-765.

-38 Davies JM, Lewis MPN, Wimperis J, Rafi I, Ladhani S, Bolton-Maggs PH; British Committee for Standards in Haematology: Review of guidelines for the prevention and treatment of infection in patients with an absent or dysfunctional spleen: prepared on behalf of the British Committee for Standards in Haematology by a working party of the Haemato-Oncology Task Force. Br J Haematol 2011;155:308-317.

-39 Vianelli N, Valdrè L, Fiacchini M, de Vivo A, Gugliotta L, Catani L, Lemoli RM, Poli M, Tura S: Longterm follow-up of idiopathic thrombocytopenic purpura in 310 patients. Haematologica 2001;86: 504-509.

-40 Kojouri K, Vesely SK, Terrell DR, George JN: Splenectomy for adult patients with idiopathic thrombocytopenic purpura: a systematic review to assess long-term platelet count responses, prediction of response, and surgical complications. Blood 2004;104:2623-2634.
41 Wang T, Xu M, Ji L, Han ZC, Yang R: Splenectomy for adult chronic idiopathic thrombocytopenic purpura: experience from a single center in China. Eur J Haematol 2005:75:424-429.

42 Sarpatwari A, Provan D, Erqou S, Sobnack R, David Tai FW, Newland AC: Autologous 111 In-labelled platelet sequestration studies in patients with primary immune thrombocytopenia (ITP) prior to splenectomy: a report from the United Kingdom ITP Registry. Br J Haematol 2010;151:477-487.

43 Cuker A, Cines DB: Evidence-based mini-review: Is indium-labeled autologous platelet scanning predictive of response to splenectomy in patients with chronic immune thrombocytopenia? Hematology Am Soc Hematol Educ Program 2010;2010: 385-386.

44 Jaglowski SM, Byrd JC, Jones JA: In-hospital mortality and trends associated with splenectomy in patients with immune-mediated thrombocytopenia (ITP). Blood 2009;114:abstr 1398.

45 Gonzalez-Porras JR, Escalante F, Pardal E, Sierra M, Garcia-Frade LJ, Redondo S, Arefi M, Aguilar C, Ortega F, de Cabo E, Fisac RM, Sanz O, Esteban C, Alberca I, Sanchez-Barba M, Santos MT, Fernandez A, Gonzalez-Lopez TJ; representing the Grupo de Trombosis y Hemostasia de Castilla y León: Safety and efficacy of splenectomy in over65 year old patients with immune thrombocytopenia. Eur J Haematol 2013;91:236-241.

46 Boyle S, White RH, Brunson A, Wun T: Splenectomy and the incidence of venous thromboembolism and sepsis in patients with immune thrombocytopenia. Blood 2013;121:4782-4790.

47 Cooper N, Evangelista ML, Amadori S, Stasi R: Should rituximab be used before or after splenectomy in patients with immune thrombocytopenic purpura. Curr Opin Hematol 2007;14:642-646.

48 Godeau B, Porcher R, Fain O, Lefrère F, Fenaux P, Cheze S, Vekhoff A, Chauveheid MP, Stirnemann J, Galicier L, Bourgeois E, Haiat S, Varet B, Leporrier M, Papo T, Khellaf M, Michel M, Bierling P: Rituximab efficacy and safety in adult splenectomy candidates with chronic immune thrombocytopenic purpura - results of a prospective multicenter phase 2 study. Blood 2008;112:999-1004.

49 Kuter DJ, Bussel JB, Lyons RM, Pullarkat V, Gernsheimer TB, Senecal FM, Aledort LM, George JN, Kessler CM, Sanz MA, Liebman HA, Slovick FT, de Wolf JT, Bourgeois E, Guthrie TH Jr, Newland A. Wasser JS, Hamburg SI, Grande C, Lefrère F, Lichtin AE, Tarantino MD, Terebelo HR, Viallard JF, Cuevas FJ, Go RS, Henry DH, Redner RL, Rice L, Schipperus MR, Guo DM, Nichol JL: Efficacy of splenectomy in patients with chronic immune thrombocytopenic purpura: a double-blind randomised controlled trial. Lancet 2008;371:395-403.

50 Bussel JB, Provan D, Shamsi T, Cheng G, Psaila B, Kovaleva L, Salama A, Jenkins JM, Roychowdhury D, Mayer B, Stone N, Arning M: Effect of eltrombopag on platelet counts and bleeding during treatment of chronic idiopathic thrombocytopenic purpura: a randomized, double-blind, placebo-controlled trial. Lancet 2009;373:641-648.

51 Sarpatwari A, Bennett D, Logie JW, Shukla A, Beach KJ, Newland A, Sanderson S, Provan D: Thromboembolic events among adult patients with idiopathic thrombocytopenic purpura in the United Kingdom General Practice Research Database. Haematologica 2010;95:1167-1175.

52 Gernsheimer TB, George JN, Aledort LM, Tarantino MD, Sunkara U, Matthew Guo D, Nichol JL: Evaluation of bleeding and thrombotic events during long-term use of romiplostim in patients with chronic immune thrombocytopenia (ITP). J Thromb Haemost 2010;8:1372-1382.
53 Catala-Lopez F, Corrales I, Martin-Serrano G, et al.: Risk of thromboembolism treated with thrombopoietin receptor agonists in adult patients with thrombocytopenia: systematic review and metaanalysis of randomized controlled trials. Med Clin (Barc) 2012;139:421-429.

54 Meyer SC, Rovó A, Tsakiris DA, Scherer K, Tichelli A, Holbro A: Severe cutaneous toxicity related to eltrombopag. Br J Haematol 2013;160: 412-414.

55 Feudjo-Tepie MA, Hall SA, Logie JW, Robinson NJ, Bennett D: Risk of cataracts among idiopathic thrombocytopenic purpura (ITP) patients in the UK General Practice Research Database (GPRD). Blood 2007;110(suppl 1 Pt 2):48b, abstr 3922.

56 Brynes RK, Orazi A, Wong RSM, Bakshi K, Bailey CK, Brainsky A: A longitudinal prospective study evaluating the effects of eltrombopag treatment on bone marrow in patients with chronic immune thrombocytopenia: interim analysis at 1 year. Blood 2012;120:abstr 2195.

57 Ghanima W, Junker P, Hasselbalch HC, Boiocchi L, Geyer JT, Feng X, Gudbrandsdottir S, Orazi A, Bussel JB: Fibroproliferative activity in patients with immune thrombocytopenia (ITP) treated with thrombopoietic agents. Br J Haematol 2011; 155:248-255.

58 Cines DB, Gernsheimer TB, Wasser J, Godeau B, Provan A, Lyons RM, Altomare I, Wang X, Woodard P: Integrated analysis of long term safety in patients (pts) with chronic immune thrombocytopenia (ITP) treated with romiplostim. Blood 2012;120:abstr 2185.

59 Jawa V, Hokom M, Hu Z, El-Abaadi N, Zhuang Y, Berger D, Gupta S, Swanson SJ, Chirmule N: Low immunogenicity of romiplostim in clinical studies with ITP subjects. Ann Hematol 2010;89(suppl): $75-85$.

60 Bussel JB, Saleh MN, Vasey SY, Mayer B, Arning M, Stone NL: Repeated short-term use of eltrombopag in patients with chronic immune thrombocytopenia (ITP). Br J Haematol 2013;160:538-546.

61 Saleh MN, Bussel JB, Cheng G, Meyer O, Bailey CK, Arning M, Brainsky A; on behalf of the EXTEND Study Group: Safety and efficacy of eltrombopag for treatment of chronic immune thrombocytopenia: results of the long-term, open-label EXTEND study. Blood 2013;121:537-545.

62 Saleh MN, Cheng G, Bussel JB, Burgess P, Bakshi $\mathrm{K}$, Brainsky A, Bailey CK: Long-term safety and efficacy of eltrombopag in adults with chronic immune thrombocytopenia (ITP): report of up to 5,5 years of treatment in EXTEND. Blood 2012;120: abstr 2198.

63 Kuter DJ, Bussel JB, Newland A, Baker RI, Lyons RM, Wasser J, Viallard JF, Macik G, Rummel M, Nie K, Jun S: Long-term treatment with romiplostim in patients with chronic immune thrombocytopenia: safety and efficacy. Br J Haematol 2013; 161:411-423.

64 Selleslag D, Janssens A, Wadenvik H, Steurer M, Quittet P, Kaiafa G, Kozak T, Papadaki H, Viallard JF, Dillingham K, Kreuzbauer G: Romiplostim for the treatment of adults with primary immune thrombocytopenia (ITP) in routine clinical practice - interim results from a large, European, observational study. Blood 2012;120:abstr 3316

65 Bussel JB, Rodeghiero F, Lyons RM, Firstenberg B, Joseph J, Kessler CM, Terriou L, Stasi R, Chang P, Sun S: Sustained hemostatic platelet counts in adults with immune thrombocytopenia (ITP) following cessation of treatment with the TPO receptor agonist romiplostim: report of 9 cases. Blood 2011;118:abstr 3281 . 
66 Leven E, Miller A, Boulad N, Haider A, Bussel JB Successful discontinuation of eltrombopag treatment in patients with chronic ITP. Blood 2012;120: abstr 1085.

67 Penalver FJ, Jiménez-Yuste V, Almagro M, Alvarez-Larrán A, Rodríguez L, Casado M, Gallur L, Giraldo P, Hernández R, Menor D, Rodríguez MJ, Caballero D, González R, Mayans J, Millán I, Cabrera JF; on behalf of the Multi-Institutional Retrospective Spanish Study on the Use of Rituximab in Refractory ITP: Rituximab in the management of chronic immune thrombocytopenic purpura: an effective and safe therapeutic alternative in refractory patients. Ann Hematol 2006;85:400-406.

68 Provan D, Butler T, Evangelista ML, Amadori S, Newland AC, Stasi R: Activity and safety profile of low-dose rituximab for the treatment of autoimmune cytopenias in adults. Haematologica 2007;92: 1695-1698.

69 Carson KR, Evens AM, Richey EA, Habermann TM, Focosi D, Seymour JF, Laubach J, Bawn SD, Gordon LI, Winter JN, Furman RR, Vose JM, Zelenetz AD, Mamtani R, Raisch DW, Dorshimer GW, Rosen ST, Muro K, Gottardi-Littell NR, Talley RL, Sartor O, Green D, Major EO, Bennett CL: Progressive multifocal leukoencephalopathy after rituximab therapy in HIV-negative patients: a report of 57 cases from the Research on Adverse Drug Events and Reports project. Blood 2009;113 4834-4840.
70 Bundesärztekammer: Querschnitts-Leitlinien (BÄK) zur Therapie mit Blutkomponenten und Plasmaderivaten, ed 4. Köln, Deutscher Ärzte-Verlag, 2009.www.baek.de/haemotherapie.

71 Greinacher A, Kiefel V, Klüter H, Kroll H, Pötzsch B, Riess H: Empfehlungen zur Thrombozytentransfusion der Thrombozyten-Arbeitsgruppe der DGTI, GTH und DGHO. Transfus Med Hemother 2006:33:528-523.

72 Grimaldi-Bensouda L, Michel M, Aubrun E, Leighton P, Viallard JF, Adoue D, Magy-Bertrand N, Tisserand G, Khellaf M, Durand JM, Quittet P, Fain O, Bonnotte B, Morin AS, Limal N, Costedoat-Chalumeau N, Morel N, Pan-Petesch B, Decaux O, Mahevas M, Ruel M, Sacre K, Lefrere F, Abenhaim L, Godeau B; PGRx Immune Thrombocytopenia Study Group: A case-control study to assess the risk of immune thrombocytopenia associated with vaccines. Blood 2012;120:4938-4944.

73 Rajantie J, Zeller B, Treutiger I, Rosthöj S; NOPHO ITP working group and five national study groups: Vaccination associated thrombocytopenic purpura in children. Vaccine 2007;25:1838-1840.

74 Gernsheimer T, James AH, Stasi R: How I treat thrombocytopenia in pregnancy. Blood 2013;121: 38-47.

75 Koyama S, Tomimatsu T, Kanagawa T, Kumasawa K, Tsutsui T, Kimura T: Reliable predictors of neonatal immune thrombocytopenia in pregnant women with idiopathic thrombocytopenic purpura. Am J Hematol 2012;87:15-21.
76 Patil AS, Dotters-Katz SK, Metjian AD, James AH, Swamy GK: Use of a thrombopoietin mimetic for chronic immune thrombocytopenic purpura in pregnancy. Obstet Gynecol 2013;122(2 Pt 2):483485.

77 Alkaabi JK, Alkindi S, Riyami NA, Zia F, Balla LM, Balla SM: Successful treatment of severe thrombocytopenia with romiplostim in a pregnant patient with systemic lupus erythematosus. Lupus 2012;21:1571-1574.

78 Moretti C, Teresa Lucciola M, Morena L, BiondiZoccai G, Laudito A, Anselmino M, Paolo Trevi G, Sheiban I: Idiopathic thrombocytopenic purpura and percutaneous coronary stenting: a dangerous duo? Int J Cardiol 2008;130:e96-e97.

79 Jubelirer SJ, Mousa L, Reddy U, Mir M, Welch CA: Coronary artery bypass grafting (CABG) in patients with immune thrombocytopenia (ITP): a community hospital experience and review of the literature. W V Med J 2011;107:10-14.

80 Cantoni N, Heizmann M, Bargetzi M: Immune thrombocytopenia and anticoagulation: the role of romiplostim in the early treatment. Br J Haematol 2012;157:639-641.

81 De La Peña A, Fareed J, Thethi I, Morales-Vidal S, Schneck MJ, Shafer D: Ischemic stroke in the setting of chronic immune thrombocytopenia in an elderly patient - a therapeutic dilemma. Clin Appl Thromb Hemost 2012;18:324-326. 